PORTIONS OF THIS REPORT ARE ILLEGIDLE. It

has been reproduced from the best available

cony to permit the broadest possible avail-

ability.

\title{
ENVIRONMENTAL MONITORING REPORT FOR PANTEX PLANT COVERING 1983
}

William A. Laseter

\section{Mason \& Hanger - Silas Mason Co., Inc. \\ Pantex Plant \\ P. O. Box 30020 \\ Amarillo, Texas 79177}

\section{Operated for the DOE \\ Under U. S. Government Contract DE-AC04-76DP00487}

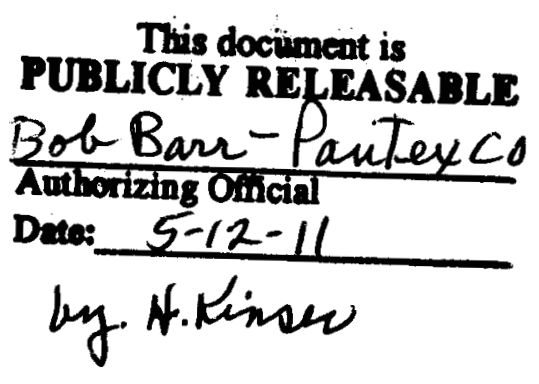

This dociunent is PUBLICLY RELDASABLE Bob Barr - PanTex Co Deriang Oincia by. H.kines

\section{DISCLAIMER}

This report was prepared as an account of work sponsored by an agency of the United States Government. Neither the United States Government nor any agency thereof, nor any of their employees, makes any warranty, express or implied, or assumes any legal liability or responsibility for the accuracy, completeness, or usefulness of any information, apparatus, product, or disclosed, or represents that its use would not infringe privately owned rights. Referproces heres, or service by trade name, trademark, ence herein to any specific commercial product, proceste or imply its endorsement, recommanufacturer, or otherwise does not necessarily constiment or any agency thereof. The views mendation, or favoring by the United States Gover secssarily state or reflect those of the and opinions of authors expressed herein do not 


\section{DISCLAIMER}

This report was prepared as an account of work sponsored by an agency of the United States Government. Neither the United States Government nor any agency Thereof, nor any of their employees, makes any warranty, express or implied, or assumes any legal liability or responsibility for the accuracy, completeness, or usefulness of any information, apparatus, product, or process disclosed, or represents that its use would not infringe privately owned rights. Reference herein to any specific commercial product, process, or service by trade name, trademark, manufacturer, or otherwise does not necessarily constitute or imply its endorsement, recommendation, or favoring by the United States Government or any agency thereof. The views and opinions of authors expressed herein do not necessarily state or reflect those of the United States Government or any agency thereof. 


\section{DISCLAIMER}

Portions of this document may be illegible in electronic image products. Images are produced from the best available original document. 
$\underline{\text { Page }}$

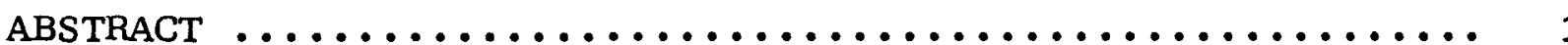

INTRODUCTION $\ldots \ldots \ldots \ldots \ldots \ldots \ldots \ldots \ldots \ldots \ldots \ldots \ldots \ldots \ldots \ldots \ldots \ldots \ldots$

ENVIRONMENTAL SETTING $\ldots \ldots \ldots \ldots \ldots \ldots \ldots \ldots \ldots \ldots \ldots \ldots \ldots \ldots$

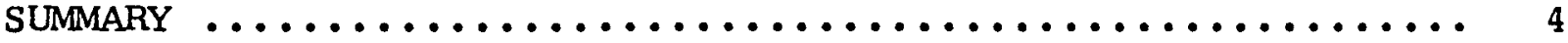

ENVIRONMENTAL MONITORING $\ldots \ldots \ldots \ldots \ldots \ldots \ldots \ldots \ldots \ldots \ldots \ldots \ldots$

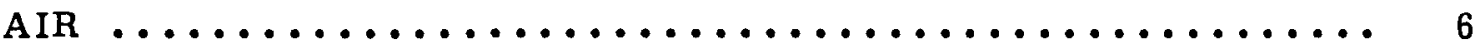

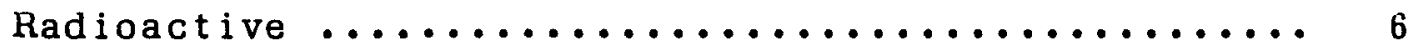

Non-Radioactive .......................... 7

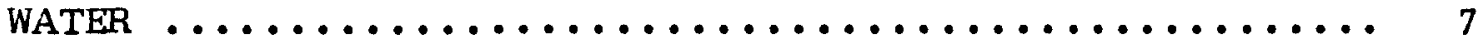

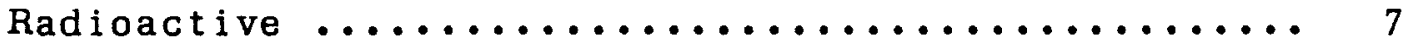

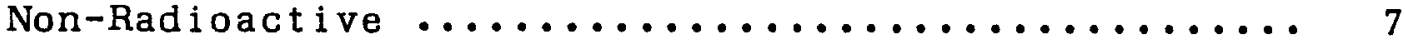

soll $\ldots \ldots \ldots \ldots \ldots \ldots \ldots \ldots \ldots \ldots \ldots \ldots \ldots \ldots \ldots \ldots \ldots \ldots \ldots \ldots \ldots$

Radioactive $\ldots \ldots \ldots \ldots \ldots \ldots \ldots \ldots \ldots \ldots \ldots \ldots \ldots \ldots \ldots$

VEGETATION $\ldots \ldots \ldots \ldots \ldots \ldots \ldots \ldots \ldots \ldots \ldots \ldots \ldots \ldots \ldots \ldots$

Radioactive $\ldots \ldots \ldots \ldots \ldots \ldots \ldots \ldots \ldots \ldots \ldots \ldots \ldots \ldots \ldots$

ВIOTA $\ldots \ldots \ldots \ldots \ldots \ldots \ldots \ldots \ldots \ldots \ldots \ldots \ldots \ldots \ldots \ldots \ldots \ldots \ldots$

Radioactive $\ldots \ldots \ldots \ldots \ldots \ldots \ldots \ldots \ldots \ldots \ldots \ldots \ldots \ldots$

CALCULATION OF POTENTIAL RADIATION DOSE TO

THE PUBLIC FROM PANTEX ACTIVITIES FOR $1983 \ldots \ldots \ldots \ldots \ldots \ldots \ldots . .9$

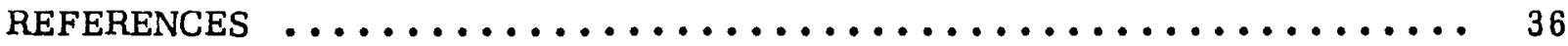

APPENDIX $\ldots \ldots \ldots \ldots \ldots \ldots \ldots \ldots \ldots \ldots \ldots \ldots \ldots \ldots \ldots \ldots \ldots \ldots \ldots \ldots \ldots$

DISTRIBUTION $\ldots \ldots \ldots \ldots \ldots \ldots \ldots \ldots \ldots \ldots \ldots \ldots \ldots \ldots \ldots \ldots \ldots \ldots$ 


\section{F I GURES}

Figure

Page

1

Physical Features of the Texas High Plains ....... 11

Pantex Plant Vicinity Map .................. 12

3

Pantex Plant Facility Site .................. 13

4

Average Winds for Amarillo, Texas for $1983 \ldots \ldots \ldots$.... 14

5 Pantex Environmental Air Sampling Network

Around an 8-Kilometer (5-Mile) Radius ........... 15

6 Environmental Soil, Water and Vegetation

Sampling Locations for Pantex Plant

(8-Kilometer [5-Mile] Radius $) \ldots \ldots \ldots \ldots \ldots \ldots$

7 Population Within an $80-\mathrm{Ki}$ lometer (50-Mile)

Radius of rantex plant ................... 17 


\begin{abstract}
This report summarizes the environmental monitoring program at Pantex Plant for 1983. It has been prepared in accordance with the United States Department of Energy Order 5484.1. This report presents monitoring data for both radioactive and non-radioactive species in the local environment.

Plant activities involve the handling of significant quantities of uranium, plutonium, and tritium. However, only small release of uranium (depleted in the isotope $\mathrm{U}-238$ ) and tritium occurred which could have affected the local environment. Monitoring data indicate that concentrations of these nuclides in the environment are below established criteria for air and water and therefore should not present a health hazard either to employees or to the public.
\end{abstract}




\section{INTRODUCTION}

Pantex Plant is a government-owned contractor-operated facility owned by the United States Department of Energy (DOE) and administered through the Amarillo Area Office. Mason \& Hanger - Silas Mason Co., Inc., is the prime operating contractor.

Pantex Plant is located in the Panhandle of Texas in Carson County. The facility is approximately 27 kilometers ( 17 miles) northeast of downtown Amarillo, and 16 kilometers ( 10 miles) west of downtown Panhandle, at $101^{\circ} 33^{\prime}$ longitude and $35^{\circ}$ $19^{\prime}$ latitude (Fig, 2). The plant site consists of 4,130 hectares $(10,200$ acres) which is a portion of the former 6,478 hectare (16,000-acre) Pantex Army Ordnance Plant which was constructed in 1942 for World War II conventional shell and bomb loading.

Operations of the Pantex Plant take place in several widely separated zones (Fig. 3). With the exception of the operating zones, and the land around them required for safety buffer or security zones, the remaining land is in native grasses and farmland. Approximately 65\% of the Plant's total area is utilized for agricultural research purposes through an agreement between Texas Tech University and the USDOE.

The principal purpose of Pantex Plant is the assembly of nuclear weapons. The overall plant mission involves the fabrication of chemical high explosive (HE) components for nuclear weapons, nuclear weapons assembly and disassembly, nuclear weapons modification and repair, and surveillance testing and disposal of chemical HE. Weapons assembly and stockpile surveillance activities involve handling significant quantities of uranium, plutonium, and tritium, as well as a variety of nonradioactive toxic chemicals.

Nuclear components are received at Pantex from other DOE plants. These components require no further processing at Pantex Plant before being assembled into weapons, thus precluding the possibility of releasing radioactive materials during normal operating conditions. Radioactive material releases at Pantex Plant are limited to certain infrequent high explosive test fires, which disperse small quantities of depleted uranium (essentially U-238), and other operations within the plant which involve the release of small amounts of tritium $(\mathrm{H}-3)$. Although not treated, airborne emissions of radioactive material (Ref. Table I) are well below prescribed standards. Likewise nonradioactive emissions (Ref. Table II) from the on-site steam generating plant are untreated but below prescribed standards.

A trickling filter sewage treatment plant for the processing of both sanitary and industrial wastes generated by the operation of the plant is located in the northeast corner of Pantex Plant. The treatment consists of primary settling, sludge digestion, trickling filter oxidation, and secondary settling. The plant processed about 500,000 gallons of water per day during 1983. After processing, the effluent is used by Texas Tech University for irrigation either by being pumped directly from the sewage plant to fields, or by being pumped to the retention playa, from which it may be pumped to the fields at a later time.

All scrap paper and food discards from the cafeteria, office areas, and manufacturing areas are bagged in plastic and buried at the Sanitary Landfill, a 3-hectare (8-acre) site surrounded by a cyclone fence. No explosives, explosive-contaminated materials, or radioactive materials are included in the landfill waste materials. All waste dumped at the landfill is covered with dirt on a daily basis. No contamination of the aquifer water below the landfill area is 
- anticipated because of the impervious topsoil and caliche layers and due to the nature of the buried materials.

Waste and excess explosives are presently destroyed by burning on open pads in accordance with approved DOE and Department of Defense (DOD) methods of disposal of explosives $(1,2)$. The explosives material, with a small amount of fuel oil added to assist in ignition, is placed on an impermeable liner on the burn pad. An explosive squib is connected from a wiring post to the waste material and the material is then squib-ignited remotely from the control bunker after personnel have cleared the area. HE-contaminated solvents are allowed to evaporate in an open tank, and the residue is burned. The burning of $\mathrm{HE}$ at Pantex Plant is approved by a written grant of authority issued by the State of Texas Air Control Board (3).

LLW (low-level radioactive waste) solid material is collected, packaged, and shipped to NTS (Nevada Test Site). This waste consists of contaminated filters and paper wipes. No liquid radiological wastes are generated or processed at Pantex Plant. During the year radioactive waste was retrieved from underground storage. This material has since been forwarded to NTS.

Chemical disposal is based on the nature of the chemical itself. Nonhazardous chemicals are buried in the sanitary landfill. Prior to burial, it is determined that the chemical will not degrade the environment and that there is no possibility of contaminating the Ogallala aquifer. Chemicals that cannot be buried or burned are shipped to an EPA-approved outside disposal agency according to current State and EPA regulations.

\section{ENVIRONMENTAL SETTING}

Pantex Plant is located between the North Central Plains and the Llano Estacado (Staked Plains) which forms part of the Great Plains (Fig. 1). The Administration Building is at an altitude of 1,078 meters $(3,538$ feet). The area is characterized by rolling grassy plains and numerous natural playas. These playas are fed only by rainwater and melted snow and are therefore frequently dry. The total precipitation in 1983 in water equivalence was $38.05 \mathrm{~cm}(14.98$ inches), as reported by the Amarillo Office of the National Weather Service. The annual evaporation rate is approximately $241 \mathrm{~cm}$ (95 inches) per year(4).

Local winds during 1983 were from the south and southwest directions approximately $40 \%$ of the time with an average annual wind velocity of 5.6 meters per second (12.6 mph) (Fig. 4). Pantex Plant is located in one of the more severe tornado areas of the United States. According to "Assessment of Tornados and Straight Wind Risks at the Pantex, Texas Site"(5), the probability of the plant site being hit by a moderate tornado ( $150 \mathrm{mph}$ rotational velocity) is approximately one in 50,000 in one year and the probability of the site being hit by a severe tornado (250 mph rotational velocity) is approximately one in $2,000,000$ in one year.

The Pantex location and surrounding area is relatively free of earthquakes. Since 1882 there have been four earthquakes in the area of the plant. These seismic events occurred on March 27, 1917, July 25, 1925, June 19, 1931, and July 20, 1966. Their epicenters were sixteen to thirty-two kilometers (ten to twenty miles) east to northeast of the plant site, and all were estimated to have intensities of $V$ or VI on the Modified Mercalli Intensity Scale(6). The Uniform Building Code places the Pantex area in Zone 1, which rates second lowest in earthquake occurrence on a scale from Zone 0 to Zone $4(7)$. 
Underlying the Texas Panhandle is the Ogallala aquifer which serves as the source of water for Pantex Plant and 35\% of the water for Amarillo. During 1983, Pantex Plant pumped 279 million gallons of water from the aquifer, and the city of Amarillo pumped 5.45 billion gallons from their Carson County field, immediately north of Pantex Plant. The saturated Ogallala aquifer is from 61 to 122 meters (200 to 400 feet) thick and at a depth below the surface of approximately 244 meters (800 feet). A surface layer of clay 18 to 24 meters (60 to 80 feet) deep forms a barrier to surface moisture and prevents deep penetration of surface contaminants $(8,9,10)$. This clay layer is usually underlaid with caliche and dry ogallala sands which cover the saturated aquifer. Aquifer water which is removed is only partially replaced through natural recharging processes.

The Panhandle area is not densely populated having approximately 255,000 persons residing within an 80 kilometer (50 mile) radius of the Pantex Plant(11). Amarillo, Texas, located southwest of the plant site, is the largest single populated center with about 150,000 residents. Pampa, Texas, located about 64 kilometers $(40$ miles) northeast of the plant, is second in population with about 21,000 residents. The remaining population is distributed throughout smaller communities, farms, and ranches. Although there is a variety of industries in the area, the local economy is influenced most significantly by agriculture and related industries.

\section{SUMMARY}

During 1983, Pantex Plant released quantities of radioactive and non-radioactive pollutants as presented in Tables I and II.

An environmental monitoring program was carried out to determine whether any Pantex effluents were having a measurable or significant effect on the environment. Calculations were performed to determine radiation dose to individuals and the public within an 80 kilometer $(50$ mile) radius. Results of these calculations show that whole body radiation dose to the public from Pantex operations was about $4 \times 10^{-8} \%$ of that due to naturally occurring external radiation.

\section{ENVIRONMENTAL MONITORING}

During 1983, Pantex Plant continued its environmental monitoring program which evaluated the collection and analyses of air, water, soil, vegetation and specific biota samples. Analyses of area samples were specific for plutonium-239, uranium, and tritium, as these are the isotopes of concern in the assembly of nuclear weapons. Analyses were also performed for radium-226 and radium-228 which occur naturally in the environment.

Isotopes common to nuclear reactor sites were not monitored at Pantex because there are no nuclear reactors or high level radioactive waste from nuclear reactors at Pantex Plant.

There were twelve off-site air sampling locations. Nine were outfitted with continuously operating Lo-volume samplers and continuously operating Hi-volume samplers. Two were outfitted with continuously operating Lo-volume samplers only, and one was out-fitted with a Hi-volume sampler.

$$
-4 \text { - }
$$


Particulate and water vapor samples were collected for radiometric analysis (Ref. Fig. 5). Air samples collected in 1983 were analyzed for total uranium, plutonium239 and tritium, and the data are presented in Tables VII through IX. In no case were any of the results in excess of the established radioactivity concentration guides (RCG's).

Water sampling was performed for ground water from Pantex wells, an off-site playa located north of the facility and for the on-site retention playa which collects all industrial and treated sanitary effluents prior to use in crop irrigation.

The data on radioactivity in water, as presented in Tables $X$ through XIV, do not indicate the presence of radioactivity discernible above natural levels.

Soil samples were collected and subjected to analysis for total uranium and plutonium-239 content at locations indicated on Fig. 6. Results are presented in Tables XV and XVI.

There are no official RCG's for radionuclides in soil. The concentrations resulting from analyses of the local soil for plutonium-239 show that local levels are indistinguishable from world-wide fallout. Total uranium analyses are also consistent with what is considered natural activity.

Analysis of vegetation to determine total uranium and tritium concentrations was performed to determine whether unusual uptake of uranium or tritium from the soil or air had occurred. As with soil, there are no established RCG's for uranium or tritium in vegetation, although RCG's for water are sometimes used for purposes of comparison.

The data in Tables XVII and XVIII are consistent with past data on vegetation and do not indicate any upward trend. Local vegetation naturally contains uranium in concentrations comparable with those in Table XVIII.

Since 1974, the jackrabbit (Lepus Californicus Gray) has been used for analysis in the environmental monitoring program. The jackrabbit was chosen as the subject because of its abundance, its prolific nature, and its habit of maintaining a limited range as long as food is available.

Three types of analyses were performed on jackrabbits as presented in Tables XIX through XXI.

The individual organ weights are provided in Table XXII to assist in the evaluation of organ doses if desired.

On the basis of the estimated quantities of uranium-238 and tritium released during 1983 (Ref. Table I) the associated average concentrations and dose equivalents were calculated for persons residing within an 80 kilometer $(50$ mile) radius of Pantex Plant. Dose calculations for the site boundary and nearest inhabitants include kidney dose as well as total body dose equivalent, since the kidney is the critical organ for uranium-238 exposure. The total body dose for all persons residing within an 80 kilometer ( 50 mile) radius of the Pantex Plant is expressed in person-rem.

Meteorological observations from the National Weather Service Office at the Amarillo Area Terminal were utilized in construction of a wind rose for the Pantex

$$
-5 \text { - }
$$


vicinity. This, together with associated climatological data, is utilized as input to a computer model for calculation of uranium and tritium concentrations within the 80 kilometer (50 mile) radius.

The computer model utilized for calculation of contaminant contributions is the Climatological Dispersion Model (CDM) written by the National Environmental Research Center of the U. S. Environmental Protection Agency(12).

The uranium and tritium concentrations in Table I were used in calculating the dose values in Table V. For the uranium calculations, separate RCG's and associated doses were used for the total body and kidney determinations since the limiting dose equivalent is different in each case.

\section{AIR}

\section{Radioactive}

Environmental air sampling is accomplished through the use of 18 continuously operating air samplers located at nine sites around an approximate 8-kilometer (5mile) radius of Pantex Plant and two continuously operating air samplers located approximately 20 kilometers $(12.5$ miles) west and southwest of the plant (Ref. Fig. 5). Each sample site on the 8-kilometer radius around Pantex Plant is outfitted with a Hi-Vol sampler and a Lo-Vol sampler. The two "background" sites south and southwest of the plant are each outfitted with a Lo-Vol sampler. In addition, a Hi-Vol sampler was stationed at the United States Department of Agriculture (USDA) Bushland Research Station, which is located about 20 kilometers (12 miles) west of Downtown Amarillo (Ref. Fig. 2). This sampler is our "background." Particulates from the filter were analyzed for plutonium-239 and total uranium. The data are presented in Tables II through VI.

The Hi-Vol samplers operate at a sampling rate of approximately 1.1 cubic metres/minute $(40 \mathrm{cfm})$. Particles are collected on an $8 \times 10$-inch filter. The filters are changed on a weekly basis, weather permitting. A weekly air sample represents about 11,400 cubic metres.

Each weekly air sample filter is composited on a monthly basis with other filters from the same air sampling station. These samples are then sent to an outside laboratory for total uranium and plutonium-239 analysis.

The Lo-Vol samples employ a silica gel column in the sampling line for collection of environmental tritium oxide. The silica gel, acting as a desiccant, removes water vapor from the air stream. Any tritium oxide present will be captured with the water vapor and is recovered for analysis. Silica gel columns are exchanged on the same frequency as the air filters.

Nominal air flow is about $42.5 \mathrm{\ell} /$ minute $(1.5 \mathrm{cfm})$. Silica gel samples are analyzed by an outside laboratory to determine tritium content.

A summary of the results for uranium, plutonium-239, and tritium in air is presented in Tables VII through IX. Deviations above background are attributed to natural meteorological distribution of worldwide fallout. During the year we change our filter material on the Hi-Vol's from Microsorban to glass fiber, which contained about $22 \times 10^{-7} \mu \mathrm{Ci}$ Uranium per filter. Our higher uranium-in-air data are attributed to this change. 


\section{Non-Radioactive}

Pantex Plant does not have any industrial processes which serve as significant sources of particulate matter, carbon monoxide, photochemical oxidants, hydrocarbons, oxides of nitrogen, or oxides of sulfur. Steam is generated by natural gas-fired boilers which are low in sulfur dioxide emissions (see Table II and Appendix, Page A-8). Other sources of pollutants are generally absent since most of the work performed is mechanical rather than chemical. Estimated emissions from Pantex Plant steam generation operations during 1983 are presented in Table II.

Some emissions occur from test fires of chemical high explosives, burning of waste high explosives, and "flashing" of high explosives-contaminated scrap metal. Air dispersion calculations indicate these releases are within applicable Federal and State Emission Regulations(13). During 1983, an estimated $200,000 \mathrm{~kg}$ of waste chemical high explosives were burned, and approximately 13,200 litres of mixtures of volatile organic solvents (toluene, acetone, methanol, etc.) and water were disposed of by evaporation.

\section{WATER}

\section{Radioactive}

Local hydrologic characteristics of the Pantex vicinity are such that there are no streams or rivers which flow through or near the plant site. Plant effluents flow into an on-site playa (designated OW-WR-08 on Fig. 6) prior to use for irrigation. A second on-site retention playa $(O W-W R-27)$ is not currently used for either collection of plant effluents or irrigation.

These on-site retention playas, a nearby natural playa ( $O W-W R-1$ ), and groundwater from plant wells are the only available locations for collecting environmental water samples. Plant wells include deep water wells into the Ogallala aquifer and two special shallow test wells designated $O W-W R-19$ and $O W-W R-20$. $O W-W R-19$ is so located to indicate whether contamination from the retention playa is leaching into the groundwater. Playa water is not considered potable as it is high in natural dissolved alkali salts. The natural playas were dry during most of 1983, which accounts for the small number of samples taken at these sampling sites.

From the sources available, water samples are collected monthly and sent to an outside laboratory for analysis of total uranium, plutonium-239, radium-226, radium -228 , and tritium content. A summary of these data for 1983 is presented in Tables $\mathrm{X}$ through XIV.

\section{Non-Radioactive}

Analyses of environmental water samples are also accomplished for nonradioactive species. The number of samples varies due to evaporation of the playas. Most of the actual analytical work is performed by an outside accredited laboratory.

A summary of analyses for nonradioactive species for 1983 is presented in Table XXIII. 


\section{Radioactive}

Thirty-two environmental soil samples were collected monthly, weather permitting, at the locations indicated in Fig. 6 according to standard soil sampling protocol. Each sample consisted of a plug from a "cookie cutter" which is 8.9 centimeters in diameter and 5.1 centimeters in depth. Each sample may also have contained a small amount of roots/vegetation.

After collection, the samples were sent to an outside laboratory, for analysis of each isotope of interest.

A summary of soil sampling results is presented in Tables XV and XVI for uranium and plutonium-239, respectively.

\section{VEGETATION}

\section{$\underline{\text { Radioactive }}$}

Environmental vegetation samples were collected from seventeen locations (Ref. Fig. 6) each month, weather permitting, for analysis of total uranium and tritium. These samples consist primarily of native and domestic grasses, although it is not uncommon for a small percentage of other vascular plants to be present in some samples.

Following collection and labeling, the samples were sent to an outside laboratory for the actual analysis. The analytical procedures used were essentially the same as for analysis of radionuclides in soil. A summary of the results is presented in Tables XVII and XVIII.

\section{BIOTA}

\section{Radioactive}

During 1983, 10 jackrabbits (Lepus Californicus Gray) were collected from within the plant perimeter and analyzed for the presence of uranium, plutonium-239, and tritium oxide. The locations where the specimens were collected were chosen carefully to help determine whether other natural levels of uranium, plutonium239 , or tritium oxide were present in the biological cycle.

Two jackrabbits were also collected from a field south of Amarillo, to be used as "controls."

Following collection, the specimens were frozen and sent to an independent laboratory for dissection and analysis. Analyses of lung, liver, kidney, bone, and muscle tissue were performed to determine the quantity of uranium, plutonium-239, and tritium present in each organ.

These rabbits were trapped in early April, following a winter of meager vegetation for food; whereas in previous years, the rabbits were trapped in October or 
November, when vegetation for food was still plentiful. Thus the small organ weights in Table XXII reflect the shortage of forage.

These data are presented in their entirety in Tables XIX through XXII. A laboratory accident resulted in loss of much of the rabbit samples. The analyses were run on very small samples; resulting in large errors and poor precision.

\section{CALCULATION OF POTENTIAL RADIATION DOSE TO THE PUBLIC FROM PANTEX ACTIVITIES FOR 1982}

Radiation exposure to the public from Pantex activities is minimal. An estimation of the overall impact to the public from routine activities was made by application of atmospheric diffusion calculations to estimated average annual radioactivity release rates using local meteorological data.

Table I provides estimated curies of depleted uranium and tritium released during 1983. These releases are considered ground level releases since the uranium and tritium were released from structures or facilities not having stacks. Average wind speed and directional frequency (Ref. Fig. 4) were determined for each of the sixteen compass points using official climatological data for Amarillo, Texas, for $1983(14)$.

Population estimates were based on figures presented in the "1980 Census of Population and Housing" for each city, town, and county within an 80-kilometer $(50-$ mile) radius of Pantex Plant $(11,15)$.

The computer model utilized in describing local atmospheric dispersion utilizes six atmospheric stability classes and six wind speed classes to describe the atmospheric characteristics of the sixteen wind sectors contained within the 80kilometer (50-mile) radius. Meteorological parameters were chosen from data obtained at the Amarillo Office of the National Weather Service and utilized as input parameters for the Climatological Dispersion Model (CDM). Following input of approximate parameters, source data, and receptor points, the CDM calculates average annual concentration of contaminants at each receptor point.

Estimated dose equivalents from the inhalation of depleted uranium and tritium were evaluated according to equation (1) which uses a simple ratio of the calculated concentration to the appropriate RCG as the basis for dose determination:

$$
D_{x}=\frac{x \cdot D}{R C G}
$$

where,

$$
\begin{aligned}
D_{X} & =\text { dose to critical organ (rem) } \\
X & =\text { concentration of nuclide (Ref. Table IV) } \\
R C G & =\text { RCG for uncontrolled area (curies/cubic metre) (16) } \\
D & =\text { Dose to critical organ associated with the appropriate RCG(16) }
\end{aligned}
$$


For Pantex operations, three groups of receptors were chosen in performing dose calculations: (a) hypothetical site boundary receptors, (b) nearest inhabitants to the plant reservation, and (c) cities, towns, and communities within an 80kilometer $(50-$ mile) radius of Pantex Plant. Dose estimates are expressed in rem or person-rem where the term "person-rem" refers to the product of dose times population for the various receptor groups.

The 80 -kilometer (50-mile) person-rem is determined according to contaminant concentration and population in sixteen sectors (Ref. Fig. 7 ) as described by equation (2):

$$
D_{80}=\sum_{N=1}^{K} \frac{{ }_{n}{ }^{D}{ }_{w b} P_{n}}{R C G_{w b}}
$$

where,

$$
\begin{aligned}
D_{80}= & 80-k i l o m e t e r \text { total body dose in person-rem } \\
x_{n}= & \begin{array}{l}
\text { average concentration for each receptor location } \\
(\text { Table iV) }
\end{array} \\
\text { RCG }_{w b}= & \begin{array}{l}
\text { radioactivity concentration guide associated } \\
\text { with the whole body as the critical organ }
\end{array} \\
\mathrm{D}_{\mathrm{wb}}= & \begin{array}{l}
\text { dose to whole body associated with the } \\
\text { appropriate RCG }
\end{array} \\
\mathrm{P}_{\mathrm{n}}= & \text { population for receptor location considered } \\
& \text { (Table II }) \\
\mathrm{K}= & \text { number of receptor locations }
\end{aligned}
$$




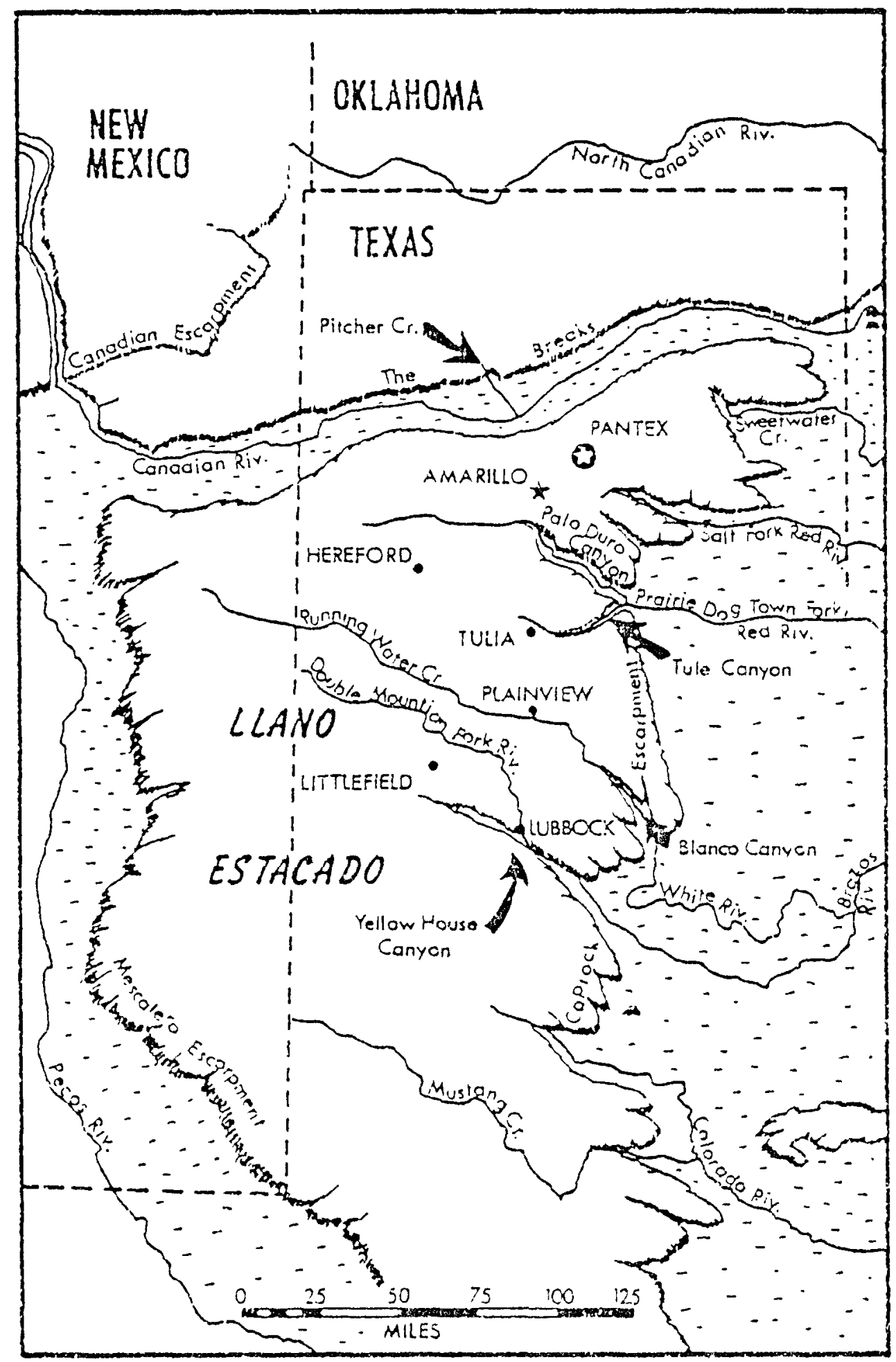

Fig. 1. Physical Fentures of the Texas itigh flains - II- 


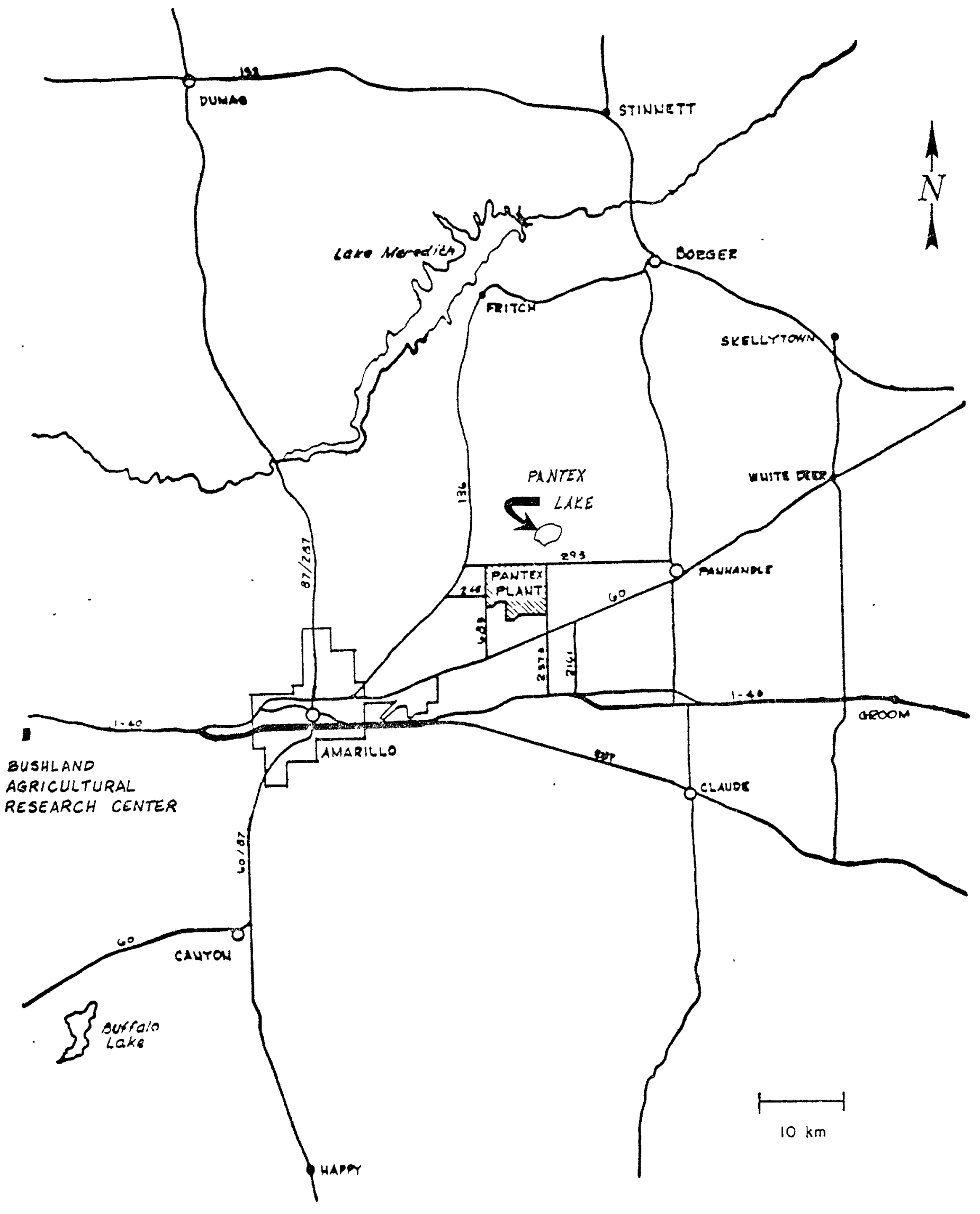

Fig. 2. Pantex Plant Vicinity Map 


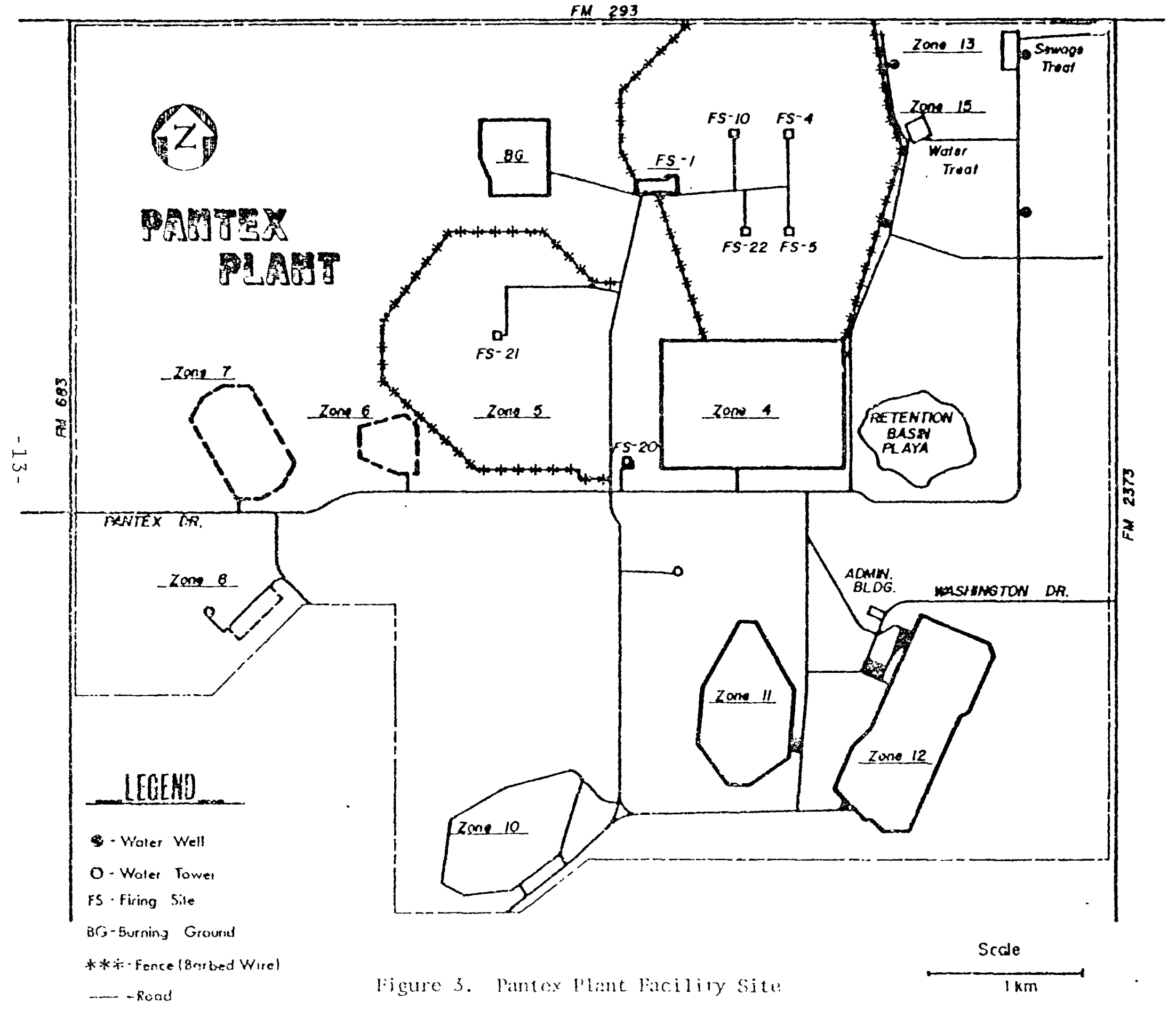




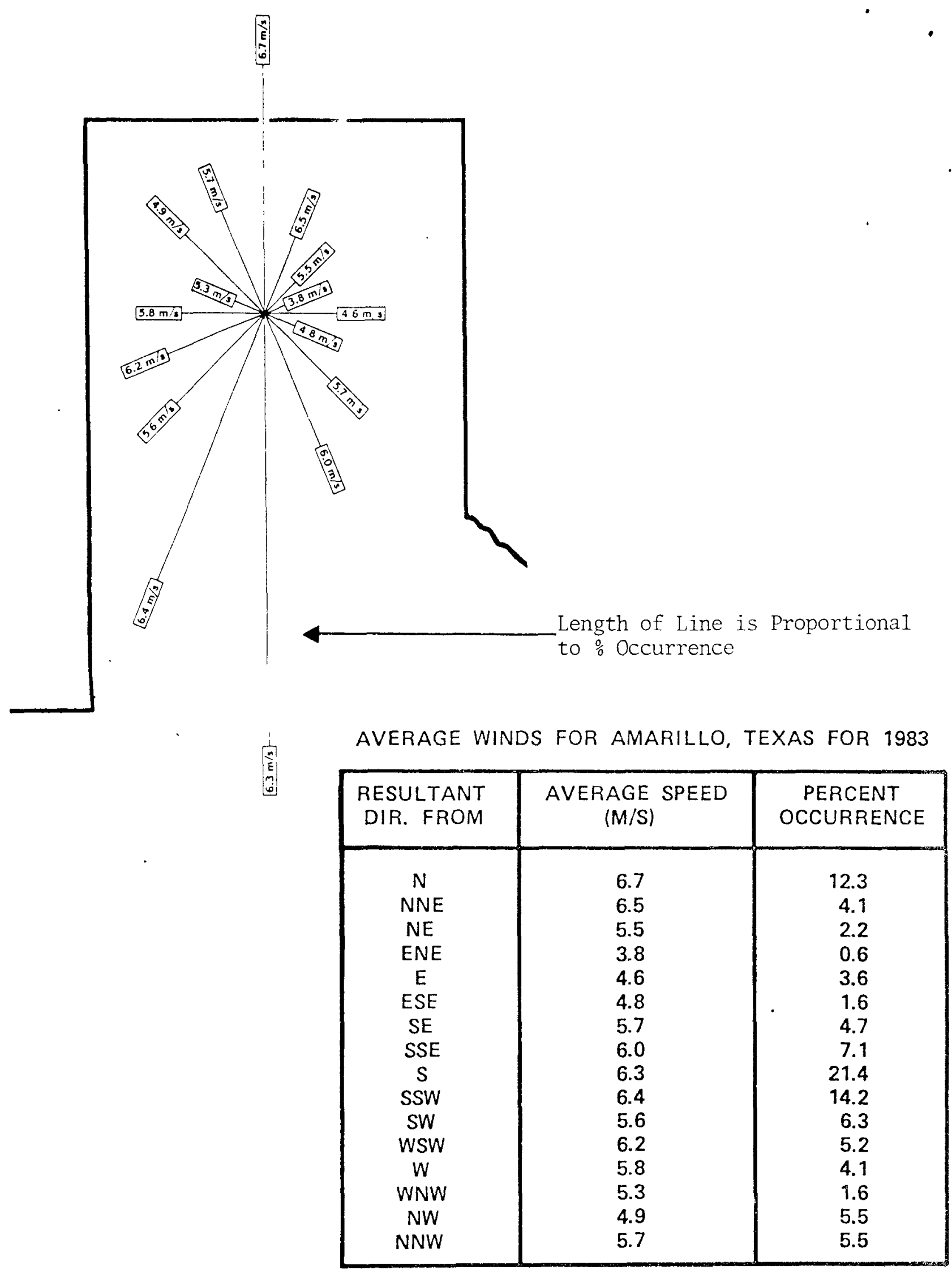

Fig. 4. Average Winds for Amari11o, Texas for 1983 


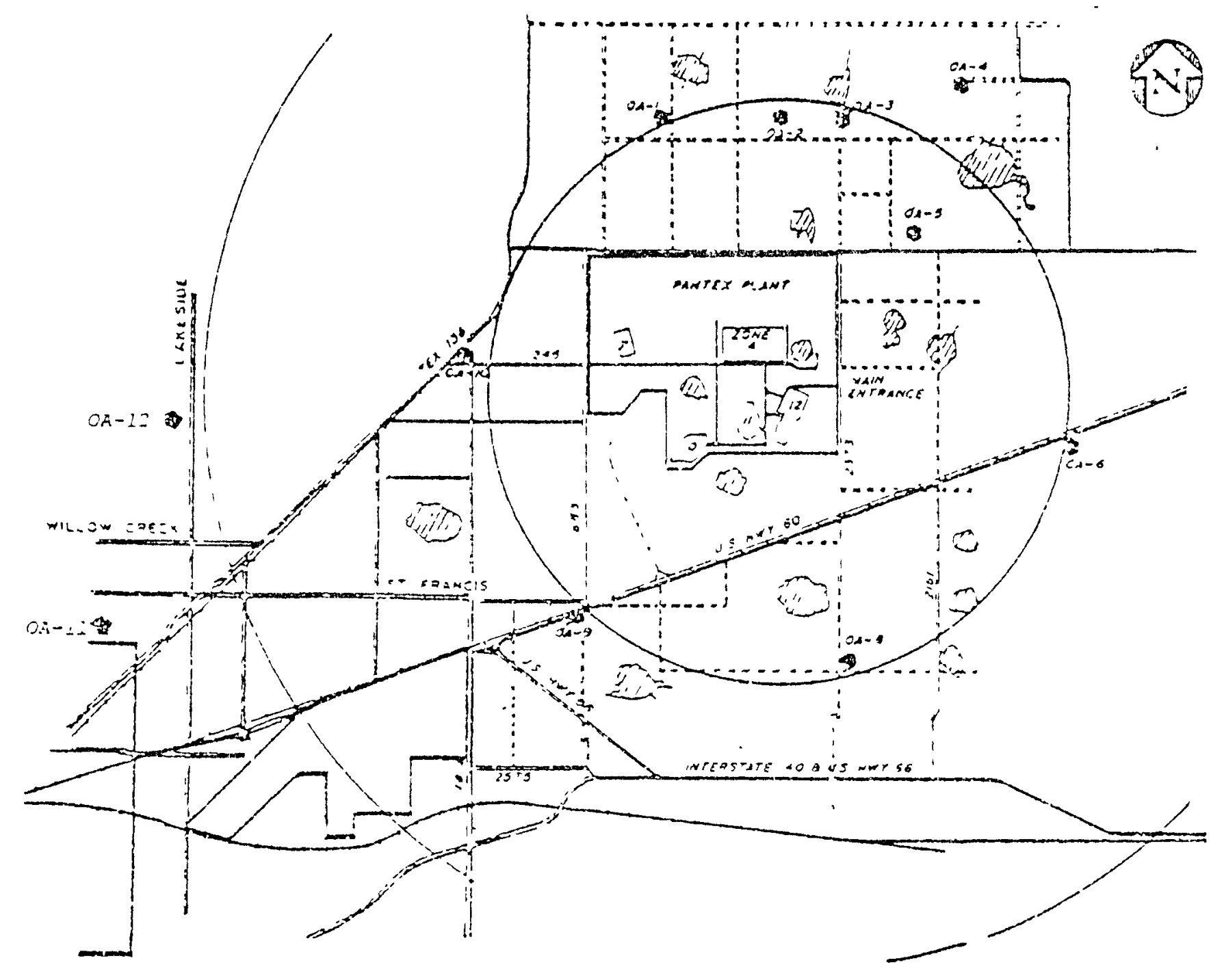

LEGEND

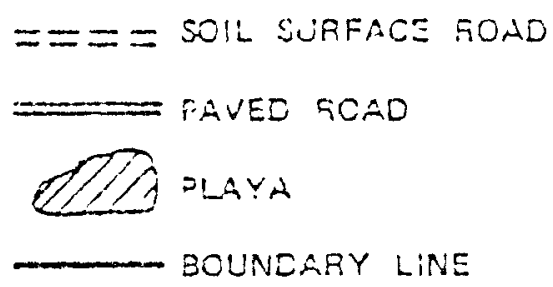

Q OFF SITE AIR SAMPLER OA

Fig. 5. Pantex Environmentui tir Sarpling Network Around on 8-Kilometer (5-Vile) Barius 


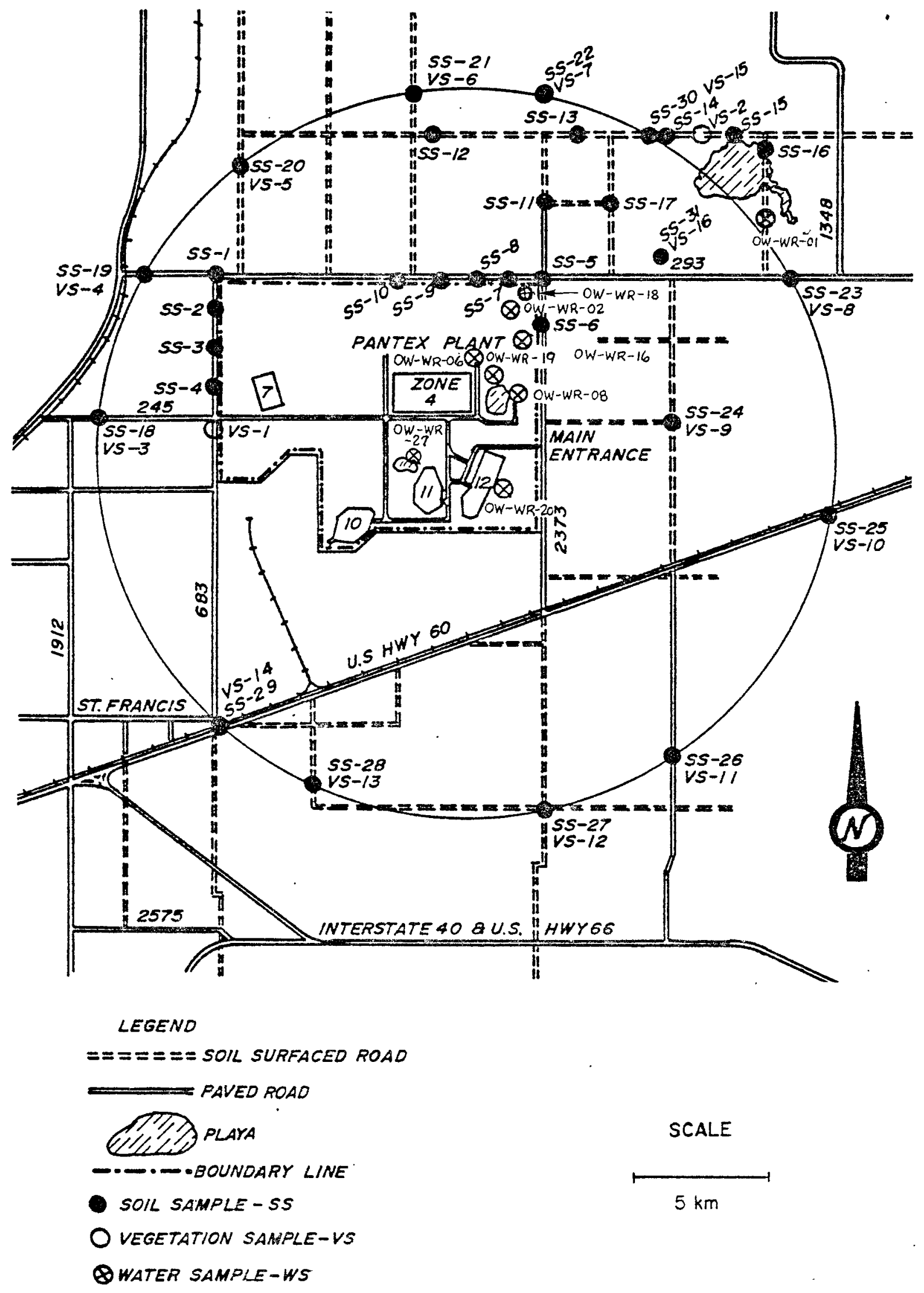

Fig. 6. Environmental Soil, Water and Vegetation Sampling Locations for Pantex Plant [8-kilometer (5-mile) Radius] 


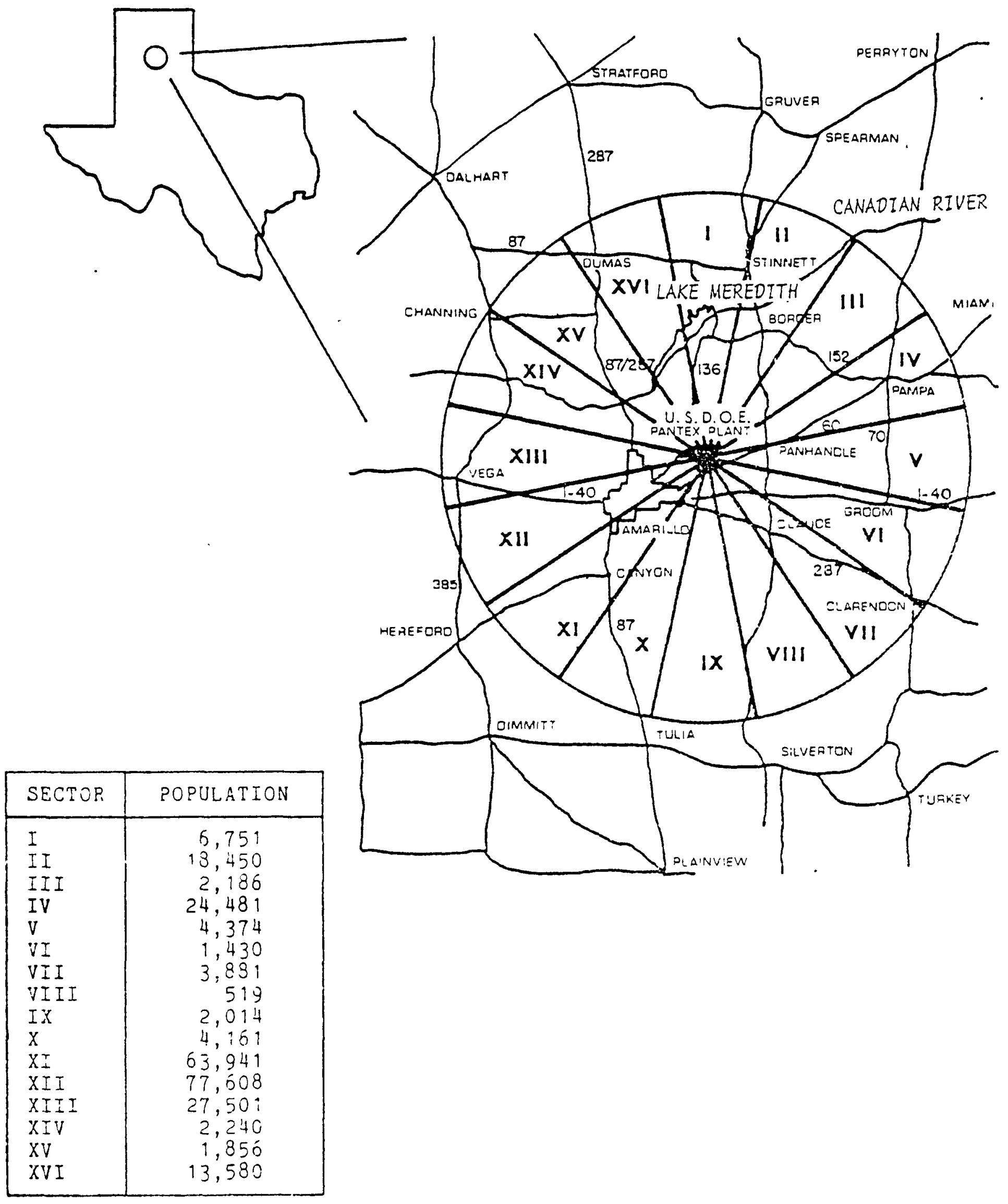

Figure 7. Population Within ar 30 Kijometer (50 Mile) Padius of Pantex Plant -17 - 


\section{FIGURES}


TABLES 


\section{TABLES}

$\underline{\text { Table }}$

Page

I Estimated Atmospheric Release of Radioactive

Material from Pantex Plant for $1983 \ldots \ldots \ldots \ldots \ldots \ldots$

I Estimated Steam Generating Plant

Emissions for $1983 \ldots \ldots \ldots \ldots \ldots \ldots \ldots \ldots \ldots \ldots \ldots \ldots$

I I Population of Area Receptors Used in 1983

Uranium and Tritium Calculations ............. 19

IV Calculated Tritium-in-Air and Uranium-in-Air

Contributions from Pantex Plant for $1983 \ldots \ldots \ldots . . .20$

V Vicinity Dose Calculations from Pantex Plant

Contributions for $1983 \ldots \ldots \ldots \ldots \ldots \ldots \ldots \ldots \ldots \ldots \ldots \ldots$

VI Pantex Vicinity Population Dose Due to

Naturally Occurring Penetrating Radiation ........ 21

VII Summary of Tritium in Air for $1983 \ldots \ldots \ldots \ldots \ldots$

VIII Summary of Plutonium 239 in Air for $1983 \ldots \ldots \ldots 2$

.IX Summary of Total Uranium in Air for $1983 \ldots \ldots \ldots$

$\mathrm{X}$ Summary of Tritium in Water for $1983 \ldots \ldots \ldots \ldots$

XI Summary of Plutonium 239 in Water for $1983 \ldots \ldots . \ldots 24$

XII Summary of Total Uranium in Water for $1983 \ldots \ldots 24$

XIII Summary of Radium 226 in Water for $1983 \ldots \ldots \ldots$

XIV Summary of Radium 228 in Water for $1983 \ldots \ldots \ldots$

XV Summary of Plutonium 239 in Soil for $1983 \ldots \ldots \ldots$

XVI Summary of Total Uranium in Soil for $1983 \ldots \ldots \ldots 27$

XVII Summary of Tritium in Vegetation for $1983 \ldots \ldots 28$

XVII Summary of Total Uranium in Vegetation for $1983 \ldots 28$

XIX Tritium in Jackrabbit for $1983 \ldots \ldots \ldots \ldots \ldots$

Xx Plutonium 239 in Jackrabbit for $1983 \ldots \ldots \ldots \ldots \ldots$

XXI Total Uranium in Jackrabbit for $1983 \ldots \ldots \ldots \ldots \ldots$

XXII Organ Weights of Jackrabbits for $1983 \ldots \ldots \ldots \ldots \ldots \ldots$

XXIII Summary of Chemical Analyses for Water

Pollutants for $1983 \ldots \ldots \ldots \ldots \ldots \ldots \ldots \ldots \ldots \ldots \ldots \ldots \ldots \ldots$ 
0

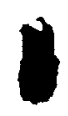

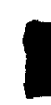

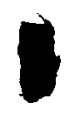


Table I. Estimated Atmospheric Releases of Radioactive Material from Pantex Plant for 1983

\begin{tabular}{|c|c|c|}
\hline Nuclide & Curies & \begin{tabular}{l}
\multicolumn{1}{c}{ Average } \\
Source Emission \\
(Curies/second)* \\
\end{tabular} \\
\hline Depleted Uranium $(U-238)$ & $1.0 \times 10^{-5}$ & $3.2 \times 10^{-13}$ \\
\hline Plutonium (Pu-239) & 0 & 0 \\
\hline Tritium $(H-3)$ & $5.0 \times 10^{-2}$ & $1.6 \times 10^{-9}$ \\
\hline
\end{tabular}

*Based on $3.15 \times 10^{7}$ seconds/year.

Table II. Est imated Steam Generating Plant Emissions for 1983

Contaminant

Carbon Monoxide

Oxides of Nitrogen
Release

$\left(1 \mathrm{~b} / 10^{6} \mathrm{Btu}\right)$

0.019

0.085 $\stackrel{\circ}{\circ}$ of $\mathrm{Standard}$

$\mathrm{N} / \mathrm{A}$

42.50

*4.2 2 1011 Btu's of gas fuel were used in 1983 .

Standard $=0.2$ pounds nitrogen $/ 10^{6} \mathrm{Btu}$ 's of gas fuel. 
Table III. Population of Area Receptors Used in 1983 Uranium and Tritium Calculations(11)

Receptor

Fritch

Sanford

Stinnett

Rural/Suburban

Borger

Rural/Suburban

Skel lytown

Rural/Suburban

Pampa

White Deer

Rural/Suburban

Panhandle

Lefors

Rural/Suburban

Groom

Rural/Suburban

$\mathrm{Cl}$ arendon

Claude

Rural/Suburban
Sector Population

I $\quad 2,299$

I 249

I $\quad 2,222$

I 1,981

II $\quad 15,834$

II $\quad 2,616$

I I I

I I I

899

1,287

IV $\quad 21,396$

IV 1,210

IV 1,875

$\begin{array}{lr}\mathrm{V} & 2,226 \\ \mathrm{~V} & 829 \\ \mathrm{~V} & 1,319\end{array}$

VI $\quad 736$

VI 694

VII

VII

VI I

2,220

1,112

549
Receptor

Rural/Suburban

Rural/Suburban

Happy

Rural/Suburban

Canyon

Amarillo

Rural/Suburban

Amarillo

Rural/Suburban

Amarillo

Vega

hural/Suburban

Channing

Rural/Suburban

Rural/Suburban
Sector Population

VI I I

519

IX 2,014

$\begin{array}{lr}X & 674 \\ X & 3,487\end{array}$

XI 10,724

XI $\quad 49,743$

XI 3,474

XI I $\quad 74,615$

XI I 2,993

XIII 24,872

XIII $\quad 900$

XI I $\quad 1,729$

Dumas XVI 12,194

Rural/Suburban XVI $\quad 1,386$ 
Table IV. Calculated Tritium-in-Air and Uranium-in-Air Contributions from Pantex Plant for 1983

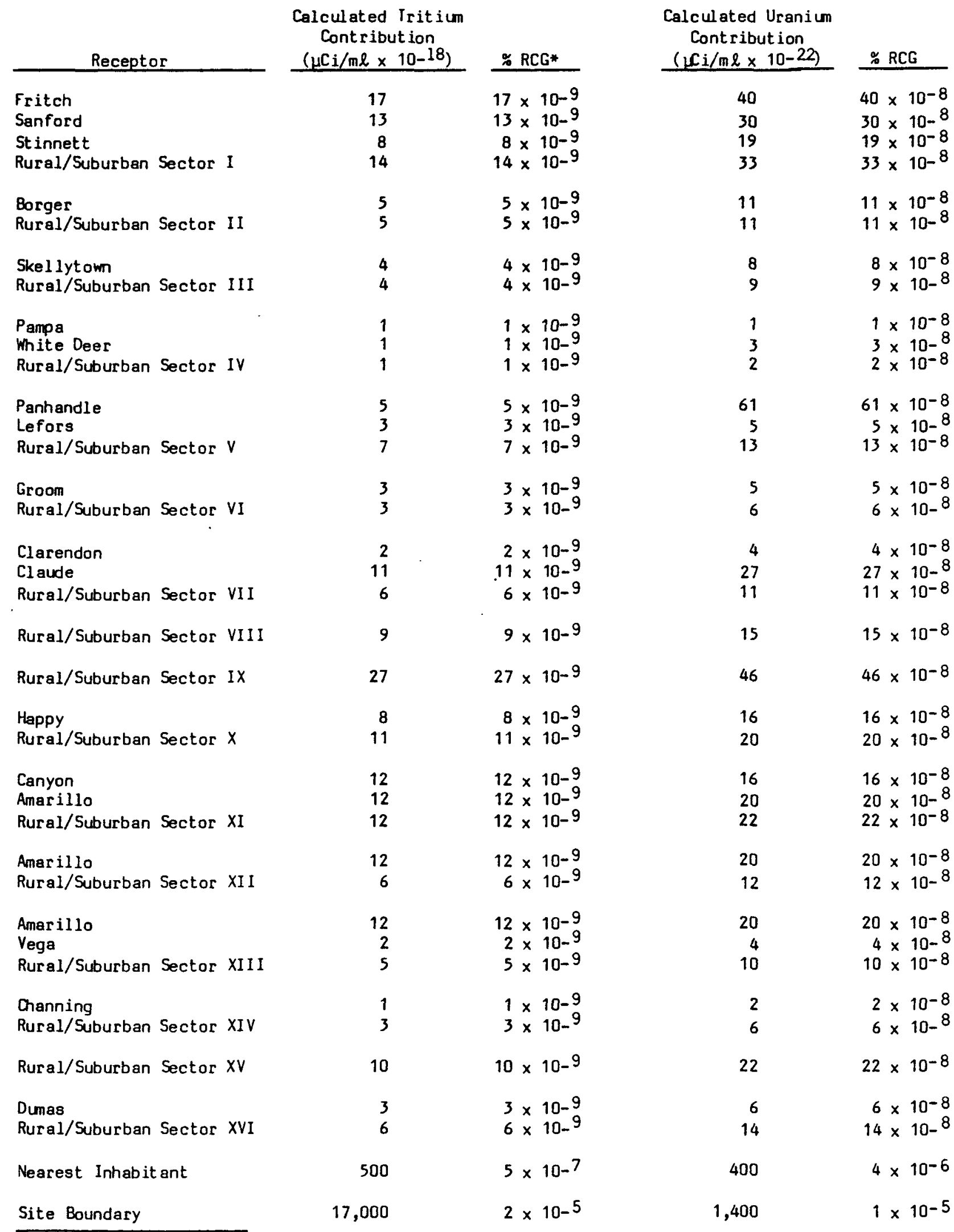

*Radioactivity Concentration Guide $=7 \times 10^{8} \mu C_{i} / \mathrm{m} \ell$ for $3_{H}$ and $1 \times 10-12 \mu C_{i} / m^{2}$ for 238 . 
Table V. Vicinity Dose Calculations from Pantex Plant Contributions for 1983*

Reference Point

Tritium ( $\mathrm{H}$ )

Uranium ( 238U)

Nearest Individual

Total Body

Kidney

Lung
$4 \times 10^{-10} \mathrm{rem}$
N/A
N/A

Site Boundary

Total Body

Kidney

Lung $\begin{array}{lll}1 \times 10-9 & \text { rem } \\ 1 \times 10-7 & \text { rem } \\ 4 \times 10^{-8} & \text { rem }\end{array}$

$\begin{array}{rr}1 \times 10^{-8} & \text { rem } \\ \text { N/A } & \\ \text { N/A } & \end{array}$

80-Ki lome ter

Total Body

$2 \times 10^{-6}$ person-rem

$4 \times 10^{-6}$ person-rem

*For Uranium-238, the kidney is the critical organ. The total body is the critical organ for tritium exposures. Total body ROG's were used in total body and person-rem determinations.

Table VI. Pantex Vicinity Population Dose Due to Naturally Occurring Penetrating Radiation*

Reference Point

80-Ki lometer

Total Body

Typical Individual

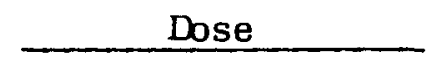

$2 \times 10^{4}$ person-rem

$8 \times 10^{-2}$ rem

*Based on an exposure rate of $9.5 \times 10^{-6} \mathrm{R} / \mathrm{hr}$ due to naturally occurring sources of penetrating radiation. 
Table VII. Summary of Tritium in Air for 1983

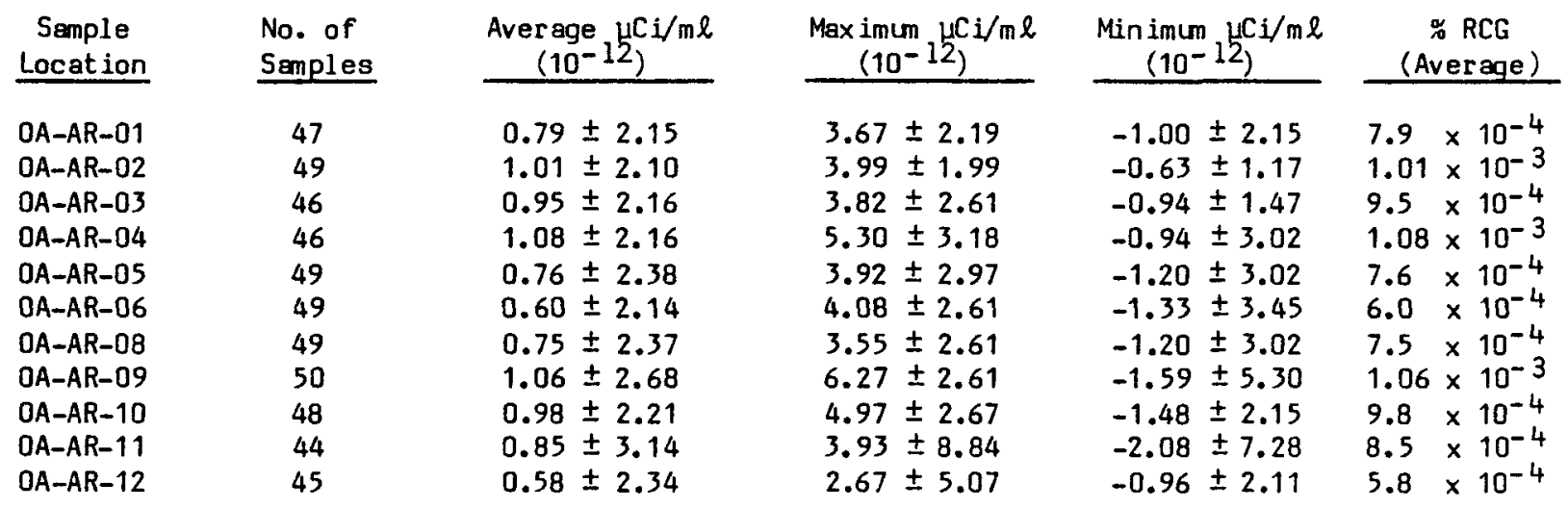

Minimum Detectable Limit $=3 \times 10^{-13} \mu \mathrm{Ci} / \mathrm{ml}$

Radioactivity Concentration Guide $=7 \times 10^{-8} \mu \mathrm{Ci} / \mathrm{ml}$

Reported errors are counting errors at the $95 \%$ confidence level

Sample locations are sited on Fig. 5. Samplers OA-AR-11 and DA-AR-12 were located near a

Southwestern Public Service Co, coal-fired power station.

Table VIII. Summary of Plutonium 239 in Air for 1983

\begin{tabular}{|c|c|c|c|c|c|}
\hline $\begin{array}{c}\text { Sample } \\
\text { Location } \\
\end{array}$ & $\begin{array}{l}\text { No. of } \\
\text { Samples } \\
\end{array}$ & $\begin{array}{c}\text { Aver age } \mu \mathrm{Ci} / \mathrm{ml} \\
\left(10^{-17}\right)\end{array}$ & $\begin{array}{c}\text { Maximum } \mu \mathrm{Ci} / \mathrm{ml} \\
\left(10^{-17}\right)\end{array}$ & $\begin{array}{c}\text { Minimum } 15 \mathrm{Ci} / \mathrm{ml} \\
\left(10^{-17}\right) \\
\end{array}$ & $\begin{array}{c}\% \text { RCG } \\
\text { (Average) }\end{array}$ \\
\hline $\begin{array}{l}0 A-A R-01 \\
O A-A R-02\end{array}$ & $\begin{array}{l}12 \\
12\end{array}$ & $\begin{array}{r}0.03 \pm 0.97 \\
<1.00 \pm 0.69\end{array}$ & $\begin{array}{l}0.95 \pm 1.11 \\
0.37 \pm 0.43\end{array}$ & $\begin{array}{l}-0.55 \pm 2.05 \\
-0.49 \pm 0.99\end{array}$ & $\begin{array}{r}1.5 \times 10^{-3} \\
<5.0 \times 10^{-2}\end{array}$ \\
\hline$O A-A R-03$ & 11 & $0.07 \pm 0.74$ & $0.99 \pm 0.99$ & $-0.29 \pm 0.42$ & $3.5 \times 10^{-3}$ \\
\hline $\begin{array}{l}\text { OA-AR-04 } \\
\text { OA-AR-05 } \\
\text { OA-AR-06 }\end{array}$ & $\begin{array}{l}12 \\
11 \\
12\end{array}$ & $\begin{array}{l}0.01 \pm 0.71 \\
0.18 \pm 0.98 \\
0.05 \pm 0.71\end{array}$ & $\begin{array}{l}0.70 \pm 0.93 \\
1.48 \pm 1.18 \\
0.89 \pm 1.09\end{array}$ & $\begin{array}{l}-0.27 \pm 0.72 \\
-0.42 \pm 0.69\end{array}$ & $\begin{array}{l}5.0 \times 10^{-4} \\
9.0 \times 10^{-3} \\
2.5 \times 10^{-3}\end{array}$ \\
\hline $\begin{array}{l}\text { OA-AR-08 } \\
\text { OA-AR-09 }\end{array}$ & $\begin{array}{l}12 \\
12\end{array}$ & $\begin{array}{l}0.15 \pm 0.83 \\
0.18 \pm 0.98\end{array}$ & $\begin{array}{l}0.71 \pm 0.87 \\
0.92 \pm 0.89\end{array}$ & $\begin{array}{l}-0.20 \pm 0.55 \\
-0.30 \pm 0.76\end{array}$ & $\begin{array}{l}7.5 \times 10^{-3} \\
9.0 \times 10^{-3}\end{array}$ \\
\hline $\begin{array}{l}\text { OA-AR-10 } \\
\text { OA-AR-13 }\end{array}$ & $\begin{array}{l}12 \\
12\end{array}$ & $\begin{array}{l}0.02 \pm 0.85 \\
0.06 \pm 0.97\end{array}$ & $\begin{array}{l}1.18 \pm 1.09 \\
1.78 \pm 1.68\end{array}$ & $\begin{array}{l}-0.40 \pm 0.45 \\
-0.39 \pm 0.69\end{array}$ & $\begin{array}{l}1.0 \times 10^{-3} \\
3.0 \times 10^{-3}\end{array}$ \\
\hline
\end{tabular}

Minimum Detectable Limit $=1 \times 10^{-17} \mu \mathrm{Ci} / \mathrm{ml}$

Radioactivity Concentration Guide $=2 \times 10^{-14} \mu \mathrm{Ci} / \mathrm{m} \ell$

Reported errors are counting errors at the $95 \%$ confidence level

Sample locations are sited on Fig. 5. Sampler OA-AR-13 was located at the agricultural research station (Figure 2). 
Table IX. Summary of Total Uranium in Air for 1983

\begin{tabular}{|c|c|c|c|c|c|}
\hline $\begin{array}{c}\text { Sample } \\
\text { Location } \\
\end{array}$ & $\begin{array}{l}\text { No. of } \\
\text { Samples }\end{array}$ & $\begin{array}{l}\text { Aver age } \mu \mathrm{Ci} / \mathrm{m} \ell \\
\left(10^{-16}\right) \\
\end{array}$ & 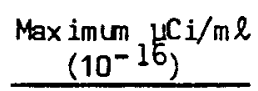 & $\begin{array}{c}\text { Minimum } 1 \mathrm{Ci} / \mathrm{m} \ell \\
\left(10^{-16}\right)\end{array}$ & $\begin{array}{c}\% \text { RCG } \\
\text { (Average) } \\
\end{array}$ \\
\hline $\begin{array}{l}\text { OA-AR-01 } \\
\text { OA-AR-02 }\end{array}$ & $\begin{array}{l}12 \\
12\end{array}$ & $\begin{array}{l}2.19 \pm 0.40 \\
2.10 \pm 0.44\end{array}$ & $\begin{array}{l}4.83 \pm 0.59 \\
4.05 \pm 0.89\end{array}$ & $\begin{array}{l}0.00 \pm 0.21 \\
0.06 \pm 0.24\end{array}$ & $\begin{array}{l}2.2 \times 10^{-3} \\
2.1 \times 10^{-3}\end{array}$ \\
\hline $\begin{array}{l}O A-A R-03 \\
O A-A R-04\end{array}$ & $\begin{array}{l}12 \\
12\end{array}$ & $\begin{array}{l}1.76 \pm 0.43 \\
1.79 \pm 0.37\end{array}$ & $\begin{array}{l}3.16 \pm 0.49 \\
3.35 \pm 0.49\end{array}$ & $\begin{array}{l}0.33 \pm 0.29 \\
0.16 \pm 0.10\end{array}$ & $\begin{array}{l}1.8 \times 10^{-3} \\
1.8 \times 10^{-3}\end{array}$ \\
\hline $\begin{array}{l}\text { OA-AR-05 } \\
\text { OA-AR-06 }\end{array}$ & $\begin{array}{l}12 \\
12\end{array}$ & $\begin{array}{l}2.21 \pm 0.41 \\
2.03 \pm 0.38\end{array}$ & $\begin{array}{l}3.55 \pm 0.49 \\
3.55 \pm 0.69\end{array}$ & $\begin{array}{l}0.55 \pm 0.28 \\
0.69 \pm 0.16\end{array}$ & $\begin{array}{l}2.2 \times 10^{-3} \\
2.0 \times 10^{-3}\end{array}$ \\
\hline $\begin{array}{l}\text { OA-AR-08 } \\
\text { OA-AR-09 }\end{array}$ & $\begin{array}{l}12 \\
12\end{array}$ & $\begin{array}{l}2.12 \pm 0.45 \\
2.22 \pm 0.43\end{array}$ & $\begin{array}{l}4.93 \pm 1.09 \\
6.22 \pm 0.99\end{array}$ & $\begin{array}{l}0.00 \pm 0.21 \\
0.99 \pm 0.30\end{array}$ & $\begin{array}{l}2.1 \times 10^{-3} \\
2.2 \times 10^{-3}\end{array}$ \\
\hline $\begin{array}{l}O A-A R-10 \\
O A-A R-13\end{array}$ & $\begin{array}{l}12 \\
12\end{array}$ & $\begin{array}{l}2.40 \pm 0.42 \\
2.65 \pm 0.50\end{array}$ & $\begin{array}{l}6.12 \pm 0.59 \\
7.50 \pm 1.18\end{array}$ & $\begin{array}{l}0.07 \pm 0.44 \\
0.51 \pm 0.26\end{array}$ & $\begin{array}{l}2.4 \times 10^{-3} \\
2.7 \times 10^{-3}\end{array}$ \\
\hline
\end{tabular}

Minimum Detectable Limit $=5 \times 10^{-18} \mu \mathrm{Ci} / \mathrm{ml}$

Radioactivity Concentration Guide $=1 \times 10^{-12} \mathrm{\mu l} / \mathrm{ml}$

Reported errors are counting errors at the $95 \%$ confidence level

Sample locations are sited on Fig. 5. Sampler OA-AR-13 was located at the Bushland Agricultural Research Station (Figure 2)

Table X. Summary of Tritiun in Water for 1983

\begin{tabular}{|c|c|c|c|c|c|}
\hline $\begin{array}{c}\text { Sample } \\
\text { Location }\end{array}$ & $\begin{array}{l}\text { No. of } \\
\text { Samples } \\
\end{array}$ & $\begin{array}{l}\text { Average } \mu \mathrm{C} i / \mathrm{m} \ell \\
\left(10^{-6}\right) \\
\end{array}$ & $\begin{array}{c}\operatorname{Maximum} \mu \mathrm{Ci} / \mathrm{ml} \\
\left(10^{-6}\right) \\
\end{array}$ & $\begin{array}{c}\text { Minimum } \mu \mathrm{Ci} / \mathrm{ml} \\
\left(10^{-6}\right) \\
\end{array}$ & $\begin{array}{c}\% \text { RCG } \\
\text { (Average) }\end{array}$ \\
\hline$O W-W R-01$ & 1 & $0.00 \pm 0.16$ & $0.00 \pm 0.16$ & $0.00 \pm 0.16$ & 0.000 \\
\hline$O W-W R-02$ & 4 & $0.12 \pm 0.20$ & $0.28 \pm 0.18$ & $0.00 \pm 0.26$ & 0.012 \\
\hline$O W-W R-06$ & 6 & $0.02 \pm 0.19$ & $0.09 \pm 0.18$ & $-0.09 \pm 0.19$ & 0.002 \\
\hline$O W-W R-0 B$ & 11 & $0.09 \pm 0.19$ & $0.38 \pm 0.18$ & $-0.03 \pm 0.26$ & 0.009 \\
\hline OW-WR-16 & 4 & $0.09 \pm 0.15$ & $0.25 \pm 0.17$ & $-0.01 \pm 0.19$ & 0.009 \\
\hline OW-WR-17 & 12 & $0.05 \pm 0.18$ & $0.34 \pm 0.18$ & $-0.10 \pm 0.17$ & 0.005 \\
\hline $0 W-W R-18$ & 6 & $0.01 \pm 0.18$ & $0.07 \pm 0.17$ & $-0.11 \pm 0.25$ & 0.001 \\
\hline OW-WR-19 & 10 & $0.11 \pm 0.19$ & $0.28 \pm 0.18$ & $-0.01 \pm 0.17$ & 0.011 \\
\hline$O W-W R-20$ & 11 & $0.01 \pm 0.19$ & $0.14 \pm 0.17$ & $-0.07 \pm 0.25$ & 0.001 \\
\hline OW-WR-23 & 12 & $0.04 \pm 0.18$ & $0.18 \pm 0.17$ & $-0.17 \pm 0.24$ & 0.004 \\
\hline$O W-W R-28$ & 11 & $<0.40 \pm 0.18$ & $0.05 \pm 0.17$ & $-0.13 \pm 0.25$ & $<0.040$ \\
\hline$O W-W R-33$ & 12 & $0.04 \pm 0.18$ & $0.21 \pm 0.17$ & $-0.13 \pm 0.16$ & 0.004 \\
\hline
\end{tabular}

Minimum Detectable Limit $=4 \times 10^{-7} \mu \mathrm{Ci} / \mathrm{ml}$

Radioactivity Concentration Guide $=1 \times 10^{-3} \mathrm{\mu i} / \mathrm{ml}$

Reported errors are counting errors at the $95 \%$ confidence level

Sample locations are sited on Fig. 6. Sample OW-WR-33 was located at the Bushland Agricultural Research Station (Figure 2) 
Table XI. Summary of Plutonium 239 in Water for 1983

\begin{tabular}{|c|c|c|c|c|c|}
\hline $\begin{array}{c}\text { Sample } \\
\text { Location } \\
\end{array}$ & $\begin{array}{l}\text { No. of } \\
\text { Samples } \\
\end{array}$ & $\begin{array}{c}\text { Average } \mu \mathrm{Ci} / \mathrm{ml} \\
\left(10^{-10}\right)\end{array}$ & $\begin{array}{c}\text { Max imum } 1 \delta^{\mathrm{C} i / m l} \\
\left(10^{-1}\right)\end{array}$ & $\begin{array}{c}\text { Minimum } \mid \mathrm{Cj} / \mathrm{m} \ell \\
\left(10^{-1}\right)\end{array}$ & $\begin{array}{c}\% \text { RCG } \\
\text { (Average) }\end{array}$ \\
\hline OW-WR-01 & 1 & $0.03 \pm 0.11$ & $0.03 \pm 0.11$ & $0.03 \pm 0.11$ & $2.0 \times 10^{-4}$ \\
\hline$O W-W R-02$ & 4 & $0.03 \pm 0.27$ & $0.17 \pm 0.13$ & $-0.03 \pm 0.08$ & $2.0 \times 10^{-4}$ \\
\hline $\begin{array}{l}\text { OW-WR-06 } \\
\text { OW-WR-08 }\end{array}$ & $\begin{array}{r}6 \\
11\end{array}$ & $\begin{array}{l}0.11 \pm 0.25 \\
0.03 \pm 0.20\end{array}$ & $\begin{array}{l}0.37 \pm 0.52 \\
0.15 \pm 0.14\end{array}$ & $\begin{array}{l}-0.07 \pm 0.10 \\
-0.05 \pm 0.10\end{array}$ & $\begin{array}{l}6.0 \times 10^{-4} \\
2.0 \times 10^{-4}\end{array}$ \\
\hline OW-WR-16 & 4 & $0.04 \pm 0.15$ & $0.21 \pm 0.12$ & $-0.02 \pm 0.08$ & $2.0 \times 10^{-4}$ \\
\hline$O W-W R-17$ & 12 & $0.07 \pm 0.22$ & $0.38 \pm 0.26$ & $-0.09 \pm 0.10$ & $4.0 \times 10^{-4}$ \\
\hline OW-WR-18 & 6 & $<0.20 \pm 0.15$ & $0.04 \pm 0.09$ & $-0.08 \pm 0.10$ & $<1.0 \times 10^{-3}$ \\
\hline OW-WR-19 & 9 & $0.03 \pm 0.16$ & $0.28 \pm 0.20$ & $-0.07 \pm 0.08$ & $2.0 \times 10^{-4}$ \\
\hline$O W-W R-20$ & 11 & $0.03 \pm 0.21$ & $0.22 \pm 0.17$ & $-0.10 \pm 0.49$ & $2.0 \times 10^{-4}$ \\
\hline$O W-W R-23$ & 12 & $0.04 \pm 0.21$ & $0.12 \pm 0.16$ & $-0.04 \pm 0.20$ & $2.0 \times 10^{-4}$ \\
\hline$O W-W R-28$ & 11 & $0.04 \pm 0.14$ & $0.20 \pm 0.12$ & $-0.04 \pm 0.16$ & $2.0 \times 10^{-4}$ \\
\hline$O W-W R-33$ & 12 & $0.02 \pm 0.20$ & $0.14 \pm 0.12$ & $-0.06 \pm 0.20$ & $1.0 \times 10^{-4}$ \\
\hline
\end{tabular}

Minimum Detectable Limit $=2 \times 10^{-11} \mu \mathrm{Ci} / \mathrm{m} \ell$

Radioactivity Concentration Guide $=2 \times 10^{-6} \mu \mathrm{Li} / \mathrm{m} \ell$

Reported errors are counting errors at the 95\% confidence level

Sample locations are sited on Fig. 6. Sample OW-WR-33 was located at the Bushland Agriculture Research Station (Figure 2)

Table XII. Summary of Total Uranium in Water for 1983

\begin{tabular}{|c|c|c|c|c|c|}
\hline $\begin{array}{l}\text { Sample } \\
\text { Location }\end{array}$ & $\begin{array}{l}\text { No. of } \\
\text { Samples }\end{array}$ & $\begin{array}{l}\text { Average } \mu \mathrm{Ci} / \mathrm{ml} \\
\left(10^{-9}\right) \\
\end{array}$ & $\frac{\operatorname{Max} \text { imum }}{\left.\left(10^{-}\right)^{C}\right)} \mathrm{i} / \mathrm{ml}$ & $\frac{\operatorname{Minimum} 1 \mathrm{HC}_{\mathrm{i} / \mathrm{ml}}}{\left(10^{-}\right)^{-}}$ & $\begin{array}{c}\text { \% RCG } \\
\text { (Average) }\end{array}$ \\
\hline OW-WR-01 & 1 & $0.80 \pm 0.40$ & $0.80 \pm 0.40$ & $0.80 \pm 0.40$ & 0.40 \\
\hline OW-WR-02 & 4 & $6.92 \pm 1.13$ & $8.00 \pm 1.30$ & $5.20 \pm 1.00$ & 3.5 \\
\hline DW-WR-06 & 6 & $6.37 \pm 1.09$ & $7.20 \pm 1.20$ & $5.70 \pm 1.10$ & 3.2 \\
\hline OW-WR-08 & 11 & $7.61 \pm 1.21$ & $9.80 \pm 1.30$ & $4.50 \pm 1.00$ & 3.8 \\
\hline$O W-W R-16$ & 4 & $6.45 \pm 1.16$ & $7.60 \pm 1.30$ & $5.70 \pm 1.00$ & 3.2 \\
\hline OW-WR-17 & 12 & $6.12 \pm 1.15$ & $13.00 \pm 2.00$ & $2.90 \pm 0.90$ & 3.1 \\
\hline$O W-W R-18$ & 6 & $5.32 \pm 1.06$ & $6.80 \pm 1.20$ & $2.00 \pm 0.60$ & 2.7 \\
\hline OW-WR-19 & 10 & $0.66 \pm 0.53$ & $3.10 \pm 0.80$ & $-0.10 \pm 0.50$ & 0.33 \\
\hline$O W-W R-20$ & 11 & $1.16 \pm 0.61$ & $3.60 \pm 0.80$ & $-0.10 \pm 0.50$ & 0.58 \\
\hline$O W-W R-23$ & 12 & $5.97 \pm 1.08$ & $7.70 \pm 1.40$ & $3.30 \pm 0.90$ & 3.0 \\
\hline$O W-W R-28$ & 11 & $6.03 \pm 1.08$ & $7.70 \pm 1.40$ & $3.80 \pm 0.90$ & 3.0 \\
\hline $0 W-W R-33$ & 12 & $4.87 \pm 0.98$ & $9.80 \pm 1.40$ & $1.60 \pm 0.60$ & 2.4 \\
\hline \multicolumn{6}{|c|}{$\begin{array}{l}\text { Minimum Detectable Limit }=3 \times 10^{-10} \mu \mathrm{Ci} / \mathrm{ml} \\
\text { Radioactivity Concentration Guide }=2 \times 10^{-7} \mu \mathrm{Ci} / \mathrm{m} \ell \\
\text { Reported errors are counting errors at the } 95 \% \text { confidence level } \\
\text { Sample locations are sited on Fig. } 6 . \text { Sample OW-WR-33 was located at the Bushland Agriculture } \\
\text { Research Station (Figure 2) }\end{array}$} \\
\hline
\end{tabular}


Table XIII. Summary of Radium 226 in Water for 1983

\begin{tabular}{|c|c|c|c|c|c|}
\hline $\begin{array}{l}\text { Sample } \\
\text { Location }\end{array}$ & $\begin{array}{l}\text { No. of } \\
\text { Samples }\end{array}$ & $\begin{array}{c}\text { Aver age } \mu \mathrm{C} \mathrm{i} / \mathrm{m} \ell \\
\left(10^{-9}\right)\end{array}$ & $\begin{array}{c}\text { Maximum } \mu C j / m \ell \\
\left(10^{-}\right)^{C}\end{array}$ & $\begin{array}{l}\text { Minimum } \mu \mathrm{Ci} / \mathrm{m} \ell \\
\left(10^{-9}\right)\end{array}$ & $\begin{array}{c}\% \text { RCG } \\
\text { (Average) }\end{array}$ \\
\hline $0 W-W R-01$ & 1 & $0.60 \pm 0.40$ & $0.60 \pm 0.40$ & $0.60 \pm 0.40$ & 6.0 \\
\hline$O W-W R-02$ & 4 & $0.27 \pm 0.48$ & $1.00 \pm 0.80$ & $-0.20 \pm 0.30$ & 2.7 \\
\hline OW-WR-06 & 6 & $0.12 \pm 0.27$ & $0.30 \pm 0.30$ & $-0.10 \pm 0.40$ & 1.2 \\
\hline $0 W-W R-08$ & 11 & $0.57 \pm 0.48$ & $1.20 \pm 0.80$ & $0.20 \pm 0.40$ & 5.7 \\
\hline$O W-W R-16$ & 4 & $0.37 \pm 0.36$ & $0.60 \pm 0.40$ & $0.10 \pm 0.40$ & 3.7 \\
\hline OW-WR-17 & 12 & $0.28 \pm 0.29$ & $0.90 \pm 0.30$ & $-0.10 \pm 0.30$ & 2.8 \\
\hline $0 W-W R-18$ & 6 & $0.23 \pm 0.29$ & $0.40 \pm 0.40$ & $0.10 \pm 0.30$ & 2.3 \\
\hline OW-WR-19 & 10 & $0.24 \pm 0.29$ & $0.60 \pm 0.40$ & $-0.10 \pm 0.20$ & 2.4 \\
\hline$O W-W R-20$ & 11 & $0.17 \pm 0.27$ & $1.20 \pm 0.50$ & $-0.20 \pm 0.30$ & 1.7 \\
\hline$O W-W R-23$ & 12 & $0.27 \pm 0.32$ & $0.80 \pm 0.50$ & $-0.10 \pm 0.20$ & 2.7 \\
\hline OW-WR-28 & 11 & $0.21 \pm 0.30$ & $0.40 \pm 0.30$ & $0.00 \pm 0.30$ & 2.1 \\
\hline$O W-W R-33$ & 12 & $0.08 \pm 0.24$ & $0.40 \pm 0.30$ & $-0.10 \pm 0.30$ & 0.8 \\
\hline
\end{tabular}

Minimum Detectable Limit $=6 \times 10^{-10} \mu \mathrm{Ci} / \mathrm{ml}$

Radioactivity Concentration Guide $=1 \times 10^{-8} \mu \mathrm{Ci} / \mathrm{ml}$

Reported errors are counting errors at the $95 \%$ confidence level

Sample locations are sited on Fig. 6. Sample OW-HR-33 was located at the Bushland Agriculture Research Station (Figure 2)

Table XIV. Summary of Radium 228 in Water for 1983

\begin{tabular}{|c|c|c|c|c|c|}
\hline $\begin{array}{c}\text { Sample } \\
\text { Location }\end{array}$ & $\begin{array}{l}\text { No. of } \\
\text { Samples }\end{array}$ & $\begin{array}{l}\text { Aver age } \mu \mathrm{Ci} / \mathrm{ml} \\
\left(10^{-9}\right)\end{array}$ & $\frac{\operatorname{Maximum}}{\left.\left(10^{-}\right)^{C}\right)}$ & $\frac{\operatorname{Minimum}}{\left.\left(10^{-}\right)^{C}\right)}$ & $\begin{array}{c}\% \text { RCG } \\
\text { (Average) }\end{array}$ \\
\hline OW-WR-01 & 1 & $0.20 \pm 1.30$ & $0.20 \pm 1.30$ & $0.20 \pm 1.30$ & 2.0 \\
\hline OW-WR-02 & 4 & $0.57 \pm 0.98$ & $1.00 \pm 1.10$ & $0.20 \pm 1.10$ & 5.7 \\
\hline $0 W-W R-06$ & 6 & $0.33 \pm 0.97$ & $1.00 \pm 1.00$ & $0.10 \pm 1.00$ & 3.3 \\
\hline OW-WR-08 & 11 & $0.56 \pm 1.40$ & $1.50 \pm 1.40$ & $-0.40 \pm 1.60$ & 5.6 \\
\hline OW-WR-16 & 4 & $0.70 \pm 1.12$ & $1.40 \pm 1.20$ & $0.20 \pm 1.10$ & 7.0 \\
\hline OW-WR-17 & 12 & $0.47 \pm 1.35$ & $1.60 \pm 1.60$ & $-1.10 \pm 2.60$ & 4.7 \\
\hline$O W-W R-18$ & 6 & $0.20 \pm 1.55$ & $1.30 \pm 1.30$ & $-1.20 \pm 2.00$ & 2.0 \\
\hline OW-WR-19 & 10 & $0.22 \pm 1.17$ & $1.60 \pm 1.00$ & $-0.80 \pm 1.60$ & 2.2 \\
\hline$O W-W R-20$ & 11 & $0.44 \pm 1.85$ & $3.60 \pm 4.90$ & $-1.50 \pm 1.70$ & 4.4 \\
\hline$O W-W R-23$ & 12 & $0.48 \pm 1.37$ & $1.50 \pm 1.60$ & $-1.20 \pm 1.90$ & 4.8 \\
\hline$O W-W R-28$ & 11 & $0.33 \pm 0.91$ & $1.20 \pm 0.90$ & $-0.40 \pm 0.80$ & 3.3 \\
\hline$O W-W R-33$ & 12 & $0.38 \pm 1.24$ & $1.10 \pm 1.40$ & $-0.60 \pm 2.20$ & 3.8 \\
\hline
\end{tabular}

Minimum Detectable Limit $=1 \times 10^{-9} \mu \mathrm{Ci} / \mathrm{ml}$

Radioactivity Concentration Guide $=1 \times 10^{-8} \mu \mathrm{Ci} / \mathrm{ml}$

Reported errors are counting errors at the $95 \%$ confidence level

Sample locations are sited on Fig. 6. Sample OW-WR-33 was located at the Bushland Agriculture Research Station (Figure 2) 
Table XV. Summary of Plutonium 239 in Soil for 1983

\begin{tabular}{|c|c|c|c|c|}
\hline Location & Samples & $\begin{array}{c}\text { Average } \mu \mathrm{Ci} / \mathrm{g} \\
\left(10^{-6}\right)\end{array}$ & $\begin{array}{l}\text { Maximum } \mu \mathrm{Ci} / \mathrm{g} \\
\left(10^{-6}\right)\end{array}$ & $\begin{array}{l}\text { Minimum } \mu C i / g \\
\left(10^{-} \sigma^{\prime}\right)\end{array}$ \\
\hline OS-SS-01 & 11 & $0.01 \pm 0.01$ & $0.02 \pm 0.01$ & $0.00 \pm 0.01$ \\
\hline $05-5 S-02$ & 11 & $0.01 \pm 0.01$ & $0.03 \pm 0.02$ & $-0.01 \pm 0.01$ \\
\hline OS-SS-03 & 11 & $0.01 \pm 0.01$ & $0.02 \pm 0.02$ & $0.00 \pm 0.01$ \\
\hline 05-5S-04 & 11 & $0.01 \pm 0.02$ & $0.04 \pm 0.02$ & $0.00 \pm 0.01$ \\
\hline OS-SS-05 & 10 & $0.01 \pm 0.01$ & $0.03 \pm 0.01$ & $0.00 \pm 0.01$ \\
\hline 0S-SS-06 & 11 & $0.01 \pm 0.01$ & $0.02 \pm 0.02$ & $0.00 \pm 0.01$ \\
\hline OS-SS-07 & 11 & $0.01 \pm 0.01$ & $0.02 \pm 0.01$ & $0.00 \pm 0.01$ \\
\hline OS-SS-08 & 11 & $0.01 \pm 0.01$ & $0.03 \pm 0.02$ & $0.00 \pm 0.01$ \\
\hline OS-SS-09 & 10 & $0.01 \pm 0.01$ & $0.02 \pm 0.01$ & $0.00 \pm 0.01$ \\
\hline $0 S-5 S-10$ & 11 & $0.01 \pm 0.01$ & $0.03 \pm 0.01$ & $0.00 \pm 0.01$ \\
\hline 0S-SS-11 & 11 & $0.01 \pm 0.01$ & $0.02 \pm 0.02$ & $-0.01 \pm 0.01$ \\
\hline OS-SS-12 & 11 & $0.00 \pm 0.01$ & $0.01 \pm 0.01$ & $-0.01 \pm 0.02$ \\
\hline OS-SS-13 & 11 & $0.01 \pm 0.01$ & $0.02 \pm 0.02$ & $-0.01 \pm 0.01$ \\
\hline OS-SS-14 & 11 & $0.01 \pm 0.01$ & $0.03 \pm 0.02$ & $0.00 \pm 0.01$ \\
\hline OS-SS-15 & 11 & $0.01 \pm 0.01$ & $0.02 \pm 0.02$ & $-0.01 \pm 0.01$ \\
\hline DS-SS-16 & 10 & $0.01 \pm 0.01$ & $0.02 \pm 0.01$ & $0.00 \pm 0.01$ \\
\hline 0S-SS-17 & 10 & $0.01 \pm 0.01$ & $0.02 \pm 0.01$ & $-0.01 \pm 0.01$ \\
\hline OS-SS-18 & 10 & $0.01 \pm 0.01$ & $0.04 \pm 0.02$ & $0.00 \pm 0.01$ \\
\hline OS-SS-19 & 11 & $0.01 \pm 0.01$ & $0.01 \pm 0.01$ & $0.00 \pm 0.01$ \\
\hline OS-SS-20 & 11 & $0.01 \pm 0.01$ & $0.02 \pm 0.01$ & $0.00 \pm 0.01$ \\
\hline OS-SS-21 & 11 & $0.00 \pm 0.01$ & $0.01 \pm 0.02$ & $0.00 \pm 0.01$ \\
\hline OS-SS-22 & 11 & $0.00 \pm 0.01$ & $0.02 \pm 0.01$ & $-0.01 \pm 0.01$ \\
\hline OS-SS-23 & 10 & $0.01 \pm 0.01$ & $0.03 \pm 0.02$ & $-0.01 \pm 0.01$ \\
\hline OS-SS-24 & 11 & $0.01 \pm 0.01$ & $0.04 \pm 0.02$ & $0.00 \pm 0.01$ \\
\hline $05-55-25$ & 11 & $0.01 \pm 0.01$ & $0.03 \pm 0.02$ & $0.00 \pm 0.00$ \\
\hline $0 S-5 S-26$ & 11 & $0.01 \pm 0.01$ & $0.04 \pm 0.02$ & $0.00 \pm 0.01$ \\
\hline $05-55-27$ & 11 & $0.01 \pm 0.01$ & $0.05 \pm 0.00$ & $0.00 \pm 0.01$ \\
\hline OS-SS-28 & 10 & $0.01 \pm 0.01$ & $0.02 \pm 0.01$ & $0.00 \pm 0.01$ \\
\hline OS-SS-29 & 11 & $0.01 \pm 0.01$ & $0.03 \pm 0.01$ & $0.00 \pm 0.01$ \\
\hline OS-SS-30 & 11 & $0.01 \pm 0.01$ & $0.02 \pm 0.01$ & $0.00 \pm 0.01$ \\
\hline OS-SS-31 & 11 & $0.01 \pm 0.01$ & $0.01 \pm 0.01$ & $0.00 \pm 0.01$ \\
\hline BU-SS-01 & 12 & $0.01 \pm 0.01$ & $0.02 \pm 0.01$ & $0.00 \pm 0.01$ \\
\hline
\end{tabular}

Minimum Detectable Limit $=2 \times 10^{-8} \mu \mathrm{Ci} / \mathrm{g}$

Reported errors are counting errors at the $95 \%$ confidence level

Activity is per gram of dry soil

Sample locations are sited on Fig. 6. Sample BU-SS-01 was located at the Bushland Agriculture Research Station (Figure 2) 
Table XVI. Summary of Total Uranium in Soil for 1983

\begin{tabular}{|c|c|c|c|c|}
\hline Location & Samples & $\begin{array}{c}\text { Average } \mu \mathrm{Ci} / \mathrm{g} \\
\left(10^{-7}\right) \\
\end{array}$ & $\begin{array}{l}\text { Maximum } \mu \mathrm{C} i / g \\
\left(10^{-7}\right) \\
\end{array}$ & $\begin{array}{l}\text { Minimum } \mu \mathrm{Ci} / \mathrm{g} \\
\quad\left(10^{-} 7\right)\end{array}$ \\
\hline OS-SS-01 & 11 & $23.09 \pm 4.32$ & $34.00 \pm 5.00$ & $11.00 \pm 3.00$ \\
\hline 0S-SS-02 & 11 & $22.55 \pm 4.32$ & $49.00 \pm 6.00$ & $13.00 \pm 4.00$ \\
\hline OS-SS-03 & 11 & $20.64 \pm 4.12$ & $34.00 \pm 5.00$ & $11.00 \pm 3.00$ \\
\hline OS-SS-04 & 11 & $23.18 \pm 4.34$ & $50.00 \pm 6.00$ & $11.00 \pm 4.00$ \\
\hline 0S-SS-05 & 11 & $20.09 \pm 4.12$ & $27.00 \pm 5.00$ & $12.00 \pm 3.00$ \\
\hline 0S-SS-06 & 11 & $19.27 \pm 3.94$ & $42.00 \pm 5.00$ & $11.00 \pm 3.00$ \\
\hline 0S-SS-07 & 11 & $18.65 \pm 3.94$ & $28.00 \pm 4.00$ & $9.10 \pm 3.00$ \\
\hline OS-SS-08 & 11 & $20.18 \pm 3.84$ & $30.00 \pm 4.00$ & $13.00 \pm 3.00$ \\
\hline $05-55-09$ & 11 & $20.64 \pm 3.92$ & $26.00 \pm 4.00$ & $14.00 \pm 3.00$ \\
\hline OS-SS-10 & 11 & $19.36 \pm 4.15$ & $32.00 \pm 5.00$ & $12.00 \pm 4.00$ \\
\hline OS-SS-11 & 11 & $21.09 \pm 3.88$ & $44.00 \pm 5.00$ & $11.00 \pm 4.00$ \\
\hline 0S-SS-12 & 11 & $18.36 \pm 3.86$ & $30.00 \pm 5.00$ & $11.00 \pm 4.00$ \\
\hline OS-SS-13 & 11 & $19.82 \pm 4.02$ & $33.00 \pm 5.00$ & $11.00 \pm 4.00$ \\
\hline OS-SS- 14 & 11 & $18.91 \pm 4.02$ & $33.00 \pm 5.00$ & $9.00 \pm 3.00$ \\
\hline OS-SS-15 & 10 & $18.10 \pm 4.08$ & $25.00 \pm 4.00$ & $10.00 \pm 3.00$ \\
\hline $0 S-5 S-16$ & 11 & $17.55 \pm 3.97$ & $26.00 \pm 5.00$ & $11.00 \pm 3.00$ \\
\hline OS-SS-17 & 10 & $18.40 \pm 4.22$ & $31.00 \pm 5.00$ & $11.00 \pm 4.00$ \\
\hline 0S-5S-18 & 11 & $18.84 \pm 4.05$ & $33.00 \pm 5.00$ & $9.20 \pm 3.00$ \\
\hline OS-SS-19 & 10 & $16.50 \pm 3.85$ & $31.00 \pm 5.00$ & $11.00 \pm 4.00$ \\
\hline OS-5S-20 & 11 & $16.91 \pm 3.78$ & $26.00 \pm 4.00$ & $8.20 \pm 2.90$ \\
\hline DS-SS-21 & 10 & $17.50 \pm 3.94$ & $26.00 \pm 4.00$ & $11.00 \pm 3.00$ \\
\hline OS-SS-22 & 11 & $19.55 \pm 3.97$ & $32.00 \pm 5.00$ & $13.00 \pm 3.00$ \\
\hline OS-SS-23 & 10 & $20.50 \pm 4.02$ & $32.00 \pm 5.00$ & $14.00 \pm 3.00$ \\
\hline $0 S-5 S-24$ & 11 & $16.91 \pm 3.84$ & $26.00 \pm 4.00$ & $11.00 \pm 3.00$ \\
\hline $0 S-5 S-25$ & 10 & $21.30 \pm 4.16$ & $33.00 \pm 5.00$ & $13.00 \pm 3.00$ \\
\hline OS-5S-26 & 10 & $19.00 \pm 3.94$ & $29.00 \pm 5.00$ & $9.00 \pm 3.10$ \\
\hline $0 S-55-27$ & 10 & $15.90 \pm 3.75$ & $23.00 \pm 4.00$ & $8.00 \pm 3.00$ \\
\hline OS-SS-28 & 11 & $17.73 \pm 4.06$ & $43.00 \pm 7.00$ & $11.00 \pm 4.00$ \\
\hline DS-SS-29 & 10 & $16.76 \pm 3.74$ & $26.00 \pm 4.00$ & $9.60 \pm 3.10$ \\
\hline OS-SS-30 & 11 & $18.91 \pm 3.75$ & $30.00 \pm 4.00$ & $12.00 \pm 4.00$ \\
\hline OS-SS-31 & 10 & $20.00 \pm 3.96$ & $29.00 \pm 4.00$ & $12.00 \pm 3.00$ \\
\hline BU-SS-01 & 11 & $19.11 \pm 3.67$ & $33.00 \pm 4.00$ & $4.20 \pm 2.60$ \\
\hline
\end{tabular}

Minimum Detectable Limit $=5 \times 10^{-8} \mu \mathrm{Ci} / \mathrm{g}$

Reported errors are counting errors at the $95 \%$ confidence level

Activity is per gran of dry soil

Sample locations are sited on Fig. 6. Sample BU-SS-01 was located in Bushland Agriculture

Research Station (Figure 2) 
Table XVII. Summary of Tritium in Vegetation for 1983

\begin{tabular}{|c|c|c|c|c|c|}
\hline $\begin{array}{c}\text { Sample } \\
\text { Location } \\
\end{array}$ & $\begin{array}{l}\text { No. of } \\
\text { Samples }\end{array}$ & $\begin{array}{l}\text { Average : } \\
\text { Moisture } \\
\end{array}$ & $\begin{array}{r}\text { Average } \mu \mathrm{Ci} / \mathrm{q} \\
\text { Mo isture }\left(10^{-6}\right) \\
\end{array}$ & $\begin{array}{r}\text { Maximum } \mu \mathrm{C} i / q \\
\text { Moisture }\left(10^{-6}\right) \\
\end{array}$ & $\begin{aligned} & \text { Minimum } \boldsymbol{C} i / q \\
& \text { Moisture }\left(10^{-b}\right. \\
&\end{aligned}$ \\
\hline OV-VS-01 & 10 & 53.0 & $0.25 \pm 0.22$ & $1.80 \pm 0.30$ & $-0.01 \pm 0.17$ \\
\hline OV-VS-02 & 11 & 64.9 & $0.13 \pm 0.18$ & $0.39 \pm 0.17$ & $-0.11 \pm 0.17$ \\
\hline OV-VS-03 & 11 & 42.0 & $0.47 \pm 0.56$ & $4.60 \pm 1.00$ & $-0.09 \pm 0.18$ \\
\hline OV-VS-04 & 10 & 46.4 & $0.02 \pm 0.49$ & $0.38 \pm 0.64$ & $-0.80 \pm 1.30$ \\
\hline OV-VS-0S & 11 & 70.7 & $<0.40 \pm 0.18$ & $0.06 \pm 0.17$ & $-0.12 \pm 0.17$ \\
\hline $0 V-V S-06$ & 11 & 54.4 & $0.14 \pm 0.22$ & $0.52 \pm 0.47$ & $0.01 \pm 0.16$ \\
\hline $0 V-V S-07$ & 11 & 54.5 & $0.15 \pm 0.30$ & $0.89 \pm 0.42$ & $-0.03 \pm 0.16$ \\
\hline OV-VS-08 & 11 & 71.5 & $0.10 \pm 0.24$ & $0.60 \pm 0.57$ & $-0.11 \pm 0.17$ \\
\hline OV-VS-09 & 11 & 54.7 & $0.07 \pm 0.19$ & $0.31 \pm 0.18$ & $-0.07 \pm 0.18$ \\
\hline ov-VS-10 & 11 & 42.4 & $0.54 \pm 0.32$ & $4.80 \pm 0.80$ & $-0.15 \pm 0.17$ \\
\hline ov-VS-11 & 11 & 53.5 & $0.11 \pm 0.26$ & $0.34 \pm 0.17$ & $-0.21 \pm 0.55$ \\
\hline ov-VS-12 & 11 & 41.4 & $0.65 \pm 0.53$ & $5.40 \pm 1.40$ & $-0.10 \pm 0.18$ \\
\hline OV-VS-13 & 11 & 48.5 & $0.43 \pm 0.37$ & $3.40 \pm 1.00$ & $-0.06 \pm 0.29$ \\
\hline OV-VS-14 & 11 & 60.1 & $0.08 \pm 0.22$ & $0.48 \pm 0.18$ & $-0.16 \pm 0.42$ \\
\hline OV-VS-15 & 11 & 69.7 & $0.07 \pm 0.19$ & $0.22 \pm 0.17$ & $-0.15 \pm 0.27$ \\
\hline OV-VS-16 & 11 & 63.8 & $0.10 \pm 0.19$ & $0.19 \pm 0.17$ & $-0.08 \pm 0.17$ \\
\hline BU-VS-01 & 12 & 52.1 & $0.04 \pm 0.49$ & $0.29 \pm 0.18$ & $-0.60 \pm 1.40$ \\
\hline
\end{tabular}

Minimum Detectable Limit $=4 \times 10^{-7} \mu \mathrm{Cj} / \mathrm{g}$ moisture

Reported errors are counting errors at the $95 \%$ confidence level

Sample locations are sited on Fig. 6. Sample BU-VS-01 was located at

the Bushland Agriculture Research Station (Figure 2)

Table XVIII. Summary of Total Uranium in Vegetation for 1983

\begin{tabular}{|c|c|c|c|c|}
\hline Location & $\begin{array}{l}\text { No. of } \\
\text { Samples } \\
\end{array}$ & $\begin{array}{c}\text { Aver age } \mu \mathrm{Ci} / g \\
\left(10^{-7}\right) \\
\end{array}$ & $\begin{array}{l}\text { Maximum } y \mathrm{Ci} / g \\
\left(10^{-} 7\right)^{-1} \\
\end{array}$ & $\begin{array}{c}\text { Minimum } f^{\mu C i / g} \\
\left(10^{-}\right)\end{array}$ \\
\hline $0 V-V S-01$ & 10 & $1.41 \pm 0.58$ & $4.50 \pm 0.80$ & $-0.13 \pm 0.37$ \\
\hline OV-VS-02 & 10 & $3.09 \pm 1.57$ & $9.80 \pm 2.30$ & $0.62 \pm 0.11$ \\
\hline OV-VS-03 & 10 & $1.71 \pm 0.60$ & $6.80 \pm 1.20$ & $0.22 \pm 0.22$ \\
\hline DV-VS-04 & 9 & $1.31 \pm 0.48$ & $5.20 \pm 0.80$ & $0.06 \pm 0.22$ \\
\hline OV-VS-05 & 10 & $2.62 \pm 1.01$ & $7.30 \pm 2.00$ & $-0.81 \pm 0.94$ \\
\hline $0 V-V S-06$ & 10 & $2.09 \pm 0.72$ & $7.90 \pm 1.40$ & $0.27 \pm 0.14$ \\
\hline OV-VS-07 & 10 & $1.06 \pm 0.43$ & $3.50 \pm 0.60$ & $0.24 \pm 0.17$ \\
\hline $0 V-V S-08$ & 10 & $2.81 \pm 0.89$ & $9.90 \pm 1.20$ & $-0.35 \pm 0.54$ \\
\hline OV-VS-09 & 10 & $2.40 \pm 1.06$ & $13.00 \pm 3.00$ & $0.16 \pm 0.29$ \\
\hline OV-VS-10 & 9 & $1.97 \pm 0.58$ & $4.80 \pm 0.60$ & $0.48 \pm 0.80$ \\
\hline$O V-V S-11$ & 10 & $1.10 \pm 0.48$ & $3.00 \pm 0.50$ & $0.43 \pm 0.33$ \\
\hline ov-VS-12 & 10 & $2.05 \pm 0.73$ & $6.20 \pm 1.20$ & $-0.05 \pm 0.18$ \\
\hline OV-VS-13 & 10 & $2.55 \pm 1.37$ & $12.00 \pm 4.00$ & $0.51 \pm 0.56$ \\
\hline $0 V-V S-14$ & 10 & $1.54 \pm 0.83$ & $3.90 \pm 1.60$ & $-0.23 \pm 0.32$ \\
\hline OV-VS-15 & 10 & $3.44 \pm 0.98$ & $16.00 \pm 2.00$ & $0.80 \pm 1.00$ \\
\hline ov-VS-16 & 10 & $3.29 \pm 1.52$ & $14.00 \pm 4.00$ & $0.17 \pm 0.52$ \\
\hline BU-VS-01 & 11 & $2.05 \pm 0.71$ & $4.60 \pm 0.90$ & $0.95 \pm 0.68$ \\
\hline
\end{tabular}

Minimum Detectable Limit $=4 \times 10^{-8} \mu \mathrm{Ci} / \mathrm{g}$

Reported errors are counting errors at the $95 \%$ confidence level

Results are per gram dry vegetation.

Sample locations are sited on Fig. 6. Sample BU-VS-01 was located at

the Bushland Agriculture Research Station (Figure 2) 
Table XIX. Tritium in Jackrabbit for 1983

\begin{tabular}{|c|c|c|c|c|c|c|c|c|}
\hline $\begin{array}{c}\text { Specimen } \\
\text { Identification } \\
\end{array}$ & $\begin{array}{c}\text { Kidney } \\
\left(10^{-6} \mu C i / g\right. \\
\text { Moisture }) \\
\end{array}$ & $\begin{array}{l}\text { Mo isture } \\
(\%) \\
\end{array}$ & $\begin{array}{c}\text { Liver } \\
\left(10^{-6} \mid \mathrm{Ci} i / g\right. \\
\text { Moisture })\end{array}$ & $\begin{array}{c}\text { Moisture } \\
\left(\begin{array}{c}(\%) \\
\%\end{array}\right) \\
\end{array}$ & 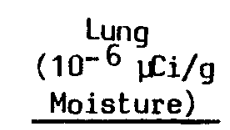 & $\begin{array}{l}\text { Moisture } \\
(\%) \\
\end{array}$ & $\begin{array}{c}\text { (Flesh) Muscle } \\
\left(10^{-6} \mu \mathrm{Ci} / \mathrm{g}\right. \\
\text { Moisture) } \\
\end{array}$ & $\begin{array}{l}\text { Moisture } \\
\text { (\%) }\end{array}$ \\
\hline Zone $12\left(\begin{array}{r}(1) \\
(2)\end{array}\right.$ & $\begin{array}{l}0.18 \pm 0.29 \\
0.06 \pm 0.26\end{array}$ & $\begin{array}{l}66.7 \\
66.7\end{array}$ & $\begin{array}{r}0.02 \pm 0.18 \\
-0.05 \pm 0.18\end{array}$ & $\begin{array}{l}63.0 \\
60.8\end{array}$ & $\begin{array}{l}0.16 \pm 0.18 \\
0.42 \pm 0.18\end{array}$ & $\begin{array}{l}68.5 \\
72.9\end{array}$ & $\begin{array}{l}0.15 \pm 0.25 \\
0.03 \pm 0.24\end{array}$ & $\begin{array}{l}37.9 \\
53.4\end{array}$ \\
\hline $\begin{array}{r}\text { Firing Site (1) } \\
(2)\end{array}$ & $\begin{array}{r}0.02 \pm 0.38 \\
-0.09 \pm 0.26\end{array}$ & $\begin{array}{l}64.8 \\
71.2\end{array}$ & $\begin{aligned}-0.12 & \pm 0.18 \\
0.00 & \pm 0.26\end{aligned}$ & $\begin{array}{l}57.8 \\
60.9\end{array}$ & $\begin{array}{l}0.19 \pm 0.18 \\
0.15 \pm 0.18\end{array}$ & $\begin{array}{l}72.5 \\
67.6\end{array}$ & $\begin{array}{l}-0.01 \pm 0.24 \\
-0.07 \pm 0.24\end{array}$ & $\begin{array}{l}79.8 \\
65.0\end{array}$ \\
\hline $\begin{array}{r}\text { Zone } 4(1) \\
(2)\end{array}$ & $\begin{array}{r}-0.12 \pm 0.26 \\
0.10 \pm 0.19\end{array}$ & $\begin{array}{l}65.4 \\
67.5\end{array}$ & $\begin{array}{l}0.05 \pm 0.19 \\
0.00 \pm 0.26\end{array}$ & $\begin{array}{l}59: 2 \\
62.1\end{array}$ & $\begin{array}{l}-0.11 \pm 0.17 \\
-0.06 \pm 0.17\end{array}$ & $\begin{array}{l}72.8 \\
71.0\end{array}$ & $\begin{array}{l}0.04 \pm 0.24 \\
0.15 \pm 0.25\end{array}$ & $\begin{array}{l}63.3 \\
56.8\end{array}$ \\
\hline $\begin{array}{cl}\text { Sewage Ireatment } & (1) \\
\text { Plant } & (2)\end{array}$ & $\begin{array}{l}0.14 \pm 0.26 \\
0.16 \pm 0.27\end{array}$ & $\begin{array}{l}63.2 \\
61.6\end{array}$ & $\begin{aligned}-0.07 & \pm 0.18 \\
0.19 & \pm 0.30\end{aligned}$ & $\begin{array}{l}55.5 \\
56.8\end{array}$ & $\begin{array}{l}0.12 \pm 0.18 \\
0.02 \pm 0.18\end{array}$ & $\begin{array}{l}72.1 \\
73.7\end{array}$ & $\begin{array}{r}0.10 \pm 0.24 \\
-0.01 \pm 0.24\end{array}$ & $\begin{array}{l}43.1 \\
51.2\end{array}$ \\
\hline $\begin{array}{ll}\text { Retention Basin (1) } \\
\text { Playa }\end{array}$ & $\begin{array}{l}0.22 \pm 0.26 \\
0.13 \pm 0.16\end{array}$ & $\begin{array}{l}50.8 \\
66.7\end{array}$ & $\begin{array}{l}-0.02 \pm 0.18 \\
-0.08 \pm 0.18\end{array}$ & $\begin{array}{l}59.5 \\
56.0\end{array}$ & $\begin{array}{r}0.09 \pm 0.18 \\
-0.06 \pm 0.17\end{array}$ & $\begin{array}{l}73.3 \\
71.6\end{array}$ & $\begin{array}{l}0.20 \pm 0.25 \\
0.07 \pm 0.24\end{array}$ & $\begin{array}{l}38.1 \\
45.7\end{array}$ \\
\hline $\begin{array}{r}\text { Control (1) } \\
\text { (2) }\end{array}$ & $\begin{array}{l}0.14 \pm 0.34 \\
0.25 \pm 0.56\end{array}$ & $\begin{array}{l}62.1 \\
43.0\end{array}$ & $\begin{array}{l}0.01 \pm 0.56 \\
0.69 \pm 0.57\end{array}$ & $\begin{array}{l}21.6 \\
16.6\end{array}$ & $\begin{array}{l}0.07 \pm 0.21 \\
0.13 \pm 0.21\end{array}$ & $\begin{array}{l}48.7 \\
63.3\end{array}$ & $\begin{array}{l}0.16 \pm 0.21 \\
0.10 \pm 0.21\end{array}$ & $\begin{array}{l}66.1 \\
61.0\end{array}$ \\
\hline
\end{tabular}

Minimum Detectable Limit $=2 \times 10^{-7} \mu \mathrm{Ci} / \mathrm{g}$ moisture. 
Table XX. Plutonium-239 in Jackrabbit for 1983

\begin{tabular}{|c|c|c|c|c|c|c|}
\hline $\begin{array}{c}\text { Specimen } \\
\text { Ident if ication } \\
\end{array}$ & $\begin{array}{c}\begin{array}{c}\text { Kidney } \\
\left(10^{-6} \mu \mathrm{Ci} / \mathrm{g}\right)\end{array} \\
\end{array}$ & $\begin{array}{c}\begin{array}{c}\text { Liver } \\
\left(10^{-6} \mu \mathrm{Ci} / \mathrm{g}\right)\end{array} \\
\end{array}$ & $\begin{array}{l}\text { Lung } \\
\left(10^{-6} \mu \mathrm{Ci} / \mathrm{g}\right)\end{array}$ & $\begin{array}{c}\text { (Flesh) Muscle } \\
\left(10^{-6} \mathrm{Ci}_{\mathrm{i} / \mathrm{g}}\right) \\
\end{array}$ & $\left(10^{-6}\right.$ & $\begin{array}{l}\text { one } \\
\left.\mathrm{L}_{\mathrm{i}} / \mathrm{q}\right)\end{array}$ \\
\hline Zone $\begin{array}{r}12(1) \\
(2)\end{array}$ & $\begin{array}{r}0.4 \pm 7.0 \\
-1.2 \pm 3.4\end{array}$ & $\begin{aligned} &-1.2 \pm 3.9 \\
& 0.6 \pm 3.0\end{aligned}$ & $\begin{array}{l}1.1 \pm 7.2 \\
0.3 \pm 3.5\end{array}$ & $\begin{array}{l}-1.2 \pm 3.0 \\
-1.4 \pm 3.9\end{array}$ & $\begin{array}{l}-0.02 \\
-0.02\end{array}$ & $\begin{array}{l} \pm 0.12 \\
\pm 0.11\end{array}$ \\
\hline $\begin{array}{r}\text { Firing Site }(1) \\
(2)\end{array}$ & $\begin{array}{l}2.6 \pm 3.6 \\
0.1 \pm 1.4\end{array}$ & $\begin{array}{l}-0.5 \pm 2.0 \\
-1.0 \pm 2.3\end{array}$ & $\begin{array}{l}-0.6 \pm 6.9 \\
-0.6 \pm 3.4\end{array}$ & $\begin{array}{l}-0.4 \pm 2.4 \\
-0.5 \pm 2.4\end{array}$ & $\begin{array}{l}-0.03 \\
-0.04\end{array}$ & $\begin{array}{l} \pm 0.11 \\
\pm 0.12\end{array}$ \\
\hline $\begin{array}{r}\text { Zone } 4(1) \\
(2)\end{array}$ & $\begin{array}{l}-0.7 \pm 3.4 \\
-0.5 \pm 2.3\end{array}$ & $\begin{array}{l}-1.4 \pm 3.9 \\
-0.3 \pm 2.4\end{array}$ & $\begin{array}{l}3.8 \pm 7.1 \\
1.1 \pm 3.5\end{array}$ & $\begin{array}{l}-0.1 \pm 3.0 \\
-0.4 \pm 2.0\end{array}$ & $\begin{array}{l}-0.06 \\
-0.05\end{array}$ & $\begin{array}{l} \pm 0.12 \\
\pm 0.11\end{array}$ \\
\hline $\begin{array}{l}\text { Sewage Treatment (1) } \\
\text { Plant }\end{array}$ & $\begin{array}{r}0.0 \pm 2.3 \\
-2.3 \pm 6.8\end{array}$ & $\begin{array}{l}-0.9 \pm 2.3 \\
-1.2 \pm 2.9\end{array}$ & $\begin{array}{l}-0.4 \pm 6.9 \\
-0.7 \pm 3.4\end{array}$ & $\begin{array}{l}-0.9 \pm 2.3 \\
-0.7 \pm 2.3\end{array}$ & $\begin{array}{l}-0.03 \\
-0.05\end{array}$ & $\begin{array}{l} \pm 0.12 \\
\pm 0.12\end{array}$ \\
\hline $\begin{array}{l}\text { Retention Basin (1) } \\
\text { Playa }\end{array}$ & $\begin{array}{r}1.0 \pm 6.9 \\
-0.9 \pm 3.4\end{array}$ & $\begin{array}{r}-0.9 \pm 2.3 \\
0.2 \pm 2.4\end{array}$ & $\begin{array}{l}-1.3 \pm 3.4 \\
-2.3 \pm 6.9\end{array}$ & $\begin{array}{l}-0.3 \pm 3.0 \\
-1.1 \pm 4.0\end{array}$ & $\begin{array}{l}0.04 \\
0.13\end{array}$ & $\begin{array}{l} \pm 0.13 \\
\pm 0.13\end{array}$ \\
\hline $\begin{array}{r}\text { Control (1) } \\
\text { (2) }\end{array}$ & * & * & * & $\begin{array}{l}0.034 \pm 0.059 \\
0.034 \pm 0.065\end{array}$ & $\begin{array}{l}0.002 \\
0.029\end{array}$ & $\begin{array}{l} \pm 0.050 \\
\pm 0.059\end{array}$ \\
\hline
\end{tabular}

Minimum Detectable Limit $=2 \times 10^{-8} \mu \mathrm{Ci} / \mathrm{g}$ Ash.

"Specimen Identification" sites are shown in Fig. 3.

* Lost in lab accident

Table XXI. Total Uranium in Jackrabbit for 1983

\begin{tabular}{|c|c|c|c|c|c|}
\hline $\begin{array}{c}\text { Specimen } \\
\text { Identification } \\
\end{array}$ & $\begin{array}{l}\text { Kidney } \\
(\mu / q \quad \text { Ash }) \\
\end{array}$ & $\begin{array}{c}\text { Liver } \\
(\mu / q \text { Ash })\end{array}$ & $\begin{array}{l}\text { Lung } \\
(\mu / 9 \text { Ash) }\end{array}$ & $\begin{array}{c}\text { (Flesh) } \\
\text { Muscle } \\
(\mu / g \quad \text { Ash }) \\
\end{array}$ & $\begin{array}{c}\text { Bone } \\
(19 / 9 \text { Ash }) \\
\end{array}$ \\
\hline $\begin{array}{r}\text { Zone } 12 \text { (1) } \\
(2)\end{array}$ & $\begin{array}{r}15.0 \\
8.6\end{array}$ & $\begin{array}{l}1.6 \\
0.6\end{array}$ & $\begin{array}{l}0.4 \\
0.4\end{array}$ & $\begin{array}{r}<0.05 \\
0.09\end{array}$ & $\begin{array}{l}0.73 \\
0.78\end{array}$ \\
\hline $\begin{array}{r}\text { Firing Site (1) } \\
\text { (2) }\end{array}$ & $\begin{array}{r}22.0 \\
1.3\end{array}$ & $\begin{array}{l}1.0 \\
4.4\end{array}$ & $\begin{array}{l}1.4 \\
0.4\end{array}$ & $\begin{array}{l}0.12 \\
0.38\end{array}$ & $\begin{array}{l}2.3 \\
0.76\end{array}$ \\
\hline $\begin{array}{r}\text { Zone } 4(1) \\
(2)\end{array}$ & $\begin{array}{l}2.0 \\
2.2\end{array}$ & $\begin{array}{l}0.4 \\
0.7\end{array}$ & $\begin{array}{l}1.0 \\
0.2\end{array}$ & $\begin{array}{r}0.05 \\
<0.05\end{array}$ & $\begin{array}{l}0.42 \\
0.66\end{array}$ \\
\hline $\begin{array}{ll}\text { Sewage Treatment } & (1) \\
\text { Plant } & (2)\end{array}$ & $\begin{array}{l}2.6 \\
6.5\end{array}$ & $\begin{array}{l}0.5 \\
0.6\end{array}$ & $\begin{array}{l}0.3 \\
0.9\end{array}$ & $\begin{array}{l}0.20 \\
0.15\end{array}$ & $\begin{array}{l}0.72 \\
0.88\end{array}$ \\
\hline $\begin{array}{l}\text { Retention Basin (1) } \\
\text { Playa }\end{array}$ & $\begin{array}{r}8.9 \\
17.0\end{array}$ & $\begin{array}{l}7.1 \\
0.5\end{array}$ & $\begin{array}{r}<0.2 \\
0.4\end{array}$ & $\begin{array}{l}0.18 \\
0.14\end{array}$ & $\begin{array}{l}0.31 \\
0.12\end{array}$ \\
\hline $\begin{array}{r}\text { Control (1) } \\
(2)\end{array}$ & $\begin{array}{l}* \\
*\end{array}$ & * & * & $\begin{array}{l}0.98 \\
1.2\end{array}$ & $\begin{array}{l}0.54 \\
1.1\end{array}$ \\
\hline
\end{tabular}


Table XXII. Organ Weights of Jackrabbits for 1983

\begin{tabular}{|c|c|c|}
\hline \multicolumn{3}{|c|}{$\begin{array}{c}\text { Specimen } \\
\text { Identification }\end{array}$} \\
\hline Zone 12 & $\begin{array}{l}(1) \\
(2)\end{array}$ & \\
\hline Firing $S$ & Site $\left(\begin{array}{l}1) \\
(2)\end{array}\right.$ & \\
\hline Zone 4 & $\begin{array}{l}(1) \\
(2)\end{array}$ & \\
\hline $\begin{array}{c}\text { Sewage } \\
\text { Plant }\end{array}$ & Treatment & $\begin{array}{l}(1) \\
(2)\end{array}$ \\
\hline $\begin{array}{c}\text { Retentio } \\
\text { Playa }\end{array}$ & on Basin & $\begin{array}{l}(1) \\
(2)\end{array}$ \\
\hline Control & $\begin{array}{l}(1) \\
(2)\end{array}$ & \\
\hline
\end{tabular}

\begin{tabular}{|c|c|c|}
\hline $\begin{array}{c}\text { K idney } \\
(g) \\
\end{array}$ & $\begin{array}{l}\text { Liver } \\
(\mathrm{g}) \\
\end{array}$ & $\begin{array}{l}\text { Lung } \\
(g) \\
\end{array}$ \\
\hline 10.8 & 20.8 & 18.4 \\
\hline 10.5 & 31.4 & 26.6 \\
\hline 10.5 & 47.4 & 16.1 \\
\hline 10.4 & 41.7 & 32.4 \\
\hline 10.4 & 28.2 & 13.6 \\
\hline 12.6 & 38.3 & $16 \cdot 2$ \\
\hline 11.4 & 41.8 & 24.4 \\
\hline 14.6 & 49.3 & 24.7 \\
\hline 12.6 & 39.0 & 22.5 \\
\hline $12 \cdot 9$ & 50.7 & 15.3 \\
\hline 13.2 & 17.6 & 14.5 \\
\hline 12.1 & 31.3 & 27.5 \\
\hline
\end{tabular}


Table XXIII. Summary of Chemical Analyses for Water Pollutants for 1983

\begin{tabular}{|c|c|c|c|c|c|c|c|}
\hline Anal ys is & $\begin{array}{c}\text { Sample } \\
\text { Identification } \\
\end{array}$ & $\begin{array}{l}\text { No. of } \\
\text { Samples } \\
\end{array}$ & Averaqe & $\frac{(\mathrm{mg} / \mathrm{l})}{\text { Max in um }}$ & Minimum & $\begin{array}{l}\text { Minimum } \\
\text { Criteria } \\
\text { (mq } / \mathrm{l}) \\
\end{array}$ & $\begin{array}{c}\text { Detectable } \\
\text { Limit } \\
\text { (mg/ l) } \\
\end{array}$ \\
\hline Silver $(A g)$ & $\begin{array}{l}\text { PL } \\
R P \\
P W \\
\text { SLP } \\
\text { BLW }\end{array}$ & $\begin{array}{r}2 \\
8 \\
30 \\
1 \\
9\end{array}$ & $\begin{array}{l}<0.01 \\
<0.01 \\
<0.01 \\
<0.01 \\
<0.01\end{array}$ & $\begin{array}{l}<0.01 \\
<0.01 \\
<0.01 \\
<0.01 \\
<0.01\end{array}$ & $\begin{array}{l}<0.01 \\
<0.01 \\
<0.01 \\
<0.01 \\
<0.01\end{array}$ & $\begin{array}{c}- \\
\overline{-} \\
0.05 \\
- \\
-\end{array}$ & $\begin{array}{l}0.01 \\
0.01 \\
0.01 \\
0.01 \\
0.01\end{array}$ \\
\hline Arsenic (As) & $\begin{array}{l}\text { PL } \\
\text { RP } \\
\text { PW } \\
\text { TW } \\
\text { STO } \\
\text { SLP } \\
\text { BUW }\end{array}$ & $\begin{array}{r}2 \\
8 \\
32 \\
15 \\
9 \\
1 \\
9\end{array}$ & $\begin{array}{l}<0.01 \\
0.012 \\
<0.01 \\
<0.01 \\
<0.011 \\
<0.01 \\
<0.01\end{array}$ & $\begin{array}{c}<0.01 \\
0.026 \\
<0.01 \\
<0.01 \\
0.018 \\
<0.01 \\
<0.01\end{array}$ & $\begin{array}{l}<0.01 \\
<0.01 \\
<0.01 \\
<0.01 \\
<0.01 \\
<0.01 \\
<0.01\end{array}$ & $\begin{array}{l}0.1 \\
0.1 \\
0.05 \\
- \\
0.1 \\
0.1 \\
-\end{array}$ & $\begin{array}{l}0.01 \\
0.01 \\
0.01 \\
0.01 \\
0.01 \\
0.01 \\
0.01\end{array}$ \\
\hline Barium (Ba) & $\begin{array}{l}\text { PL } \\
\text { RP } \\
\text { PW } \\
\text { SLP } \\
\text { BUW }\end{array}$ & $\begin{array}{r}2 \\
8 \\
32 \\
1 \\
9\end{array}$ & $\begin{array}{r}0.15 \\
0.14 \\
0.15 \\
<0.10 \\
<0.10\end{array}$ & $\begin{array}{r}0.19 \\
0.20 \\
0.29 \\
<0.10 \\
0.15\end{array}$ & $\begin{array}{r}0.10 \\
0.10 \\
0.10 \\
<0.10 \\
<0.10\end{array}$ & $\begin{array}{c}1.0 \\
1.0 \\
1.0 \\
1.0 \\
-\end{array}$ & $\begin{array}{l}0.1 \\
0.1 \\
0.1 \\
0.1 \\
0.1\end{array}$ \\
\hline Cadmium ( $\left.C_{d}\right)$ & $\begin{array}{l}\text { PL } \\
\text { RP } \\
\text { PW } \\
\text { TW } \\
\text { STD } \\
\text { SLP } \\
\text { BUW }\end{array}$ & $\begin{array}{r}2 \\
8 \\
31 \\
15 \\
9 \\
1 \\
9\end{array}$ & $\begin{array}{l}<0.001 \\
<0.002 \\
<0.002 \\
<0.001 \\
<0.002 \\
<0.001 \\
<0.0011\end{array}$ & $\begin{array}{c}<0.001 \\
0.003 \\
0.010 \\
<0.001 \\
0.008 \\
- \\
0.0013\end{array}$ & $\begin{array}{c}<0.001 \\
<0.001 \\
<0.001 \\
<0.001 \\
<0.001 \\
- \\
<0.001\end{array}$ & $\begin{array}{l}0.005 \\
0.005 \\
0.01 \\
- \\
0.005 \\
0.005 \\
-\end{array}$ & $\begin{array}{l}0.001 \\
0.001 \\
0.001 \\
0.001 \\
0.001 \\
0.001 \\
0.001\end{array}$ \\
\hline $\begin{array}{l}\text { Total } \\
\text { Chromium }\left(C_{r}\right)\end{array}$ & $\begin{array}{l}\text { PL } \\
\text { RP } \\
\text { PW } \\
\text { TW } \\
\text { STD } \\
\text { SLP } \\
\text { BUW }\end{array}$ & $\begin{array}{r}2 \\
8 \\
32 \\
14 \\
9 \\
1 \\
9\end{array}$ & $\begin{array}{l}<0.01 \\
<0.01 \\
<0.01 \\
<0.01 \\
<0.02 \\
<0.01 \\
<0.01\end{array}$ & $\begin{array}{r}<0.01 \\
<0.01 \\
<0.01 \\
<0.01 \\
0.02 \\
<0.01 \\
<0.01\end{array}$ & $\begin{array}{l}<0.01 \\
<0.01 \\
<0.01 \\
<0.01 \\
<0.01 \\
<0.01 \\
<0.01\end{array}$ & $\begin{array}{c}- \\
\overline{0} \\
0.05 \\
- \\
- \\
- \\
-\end{array}$ & $\begin{array}{l}0.01 \\
0.01 \\
0.01 \\
0.01 \\
0.01 \\
0.01 \\
0.01\end{array}$ \\
\hline
\end{tabular}

\footnotetext{
$P L$ = Pantex Lake - (OW-WR-01)

$R P=$ Retention Playa - (OW-WR-08)

PW = Potable Water - (OW-WR-02, OW-WR-06, OW-WR-16, OW-WR-18, OW-WR-23, OW-WR-28)

$T W=$ Test We1ls - (OW-WR-19, OW-WR-20)

STD = Sewage Treatment Discharge - (OW-WR-17)

SLP = Sanitary Landfill Playa (OW-WR-27)

BUW = Bushland Experimental Station Water We1l (OW-WR-33)

Sample locations are identified on Fig. 6. and Page A-9.
} 
Table XXIII. CONTINUED

\begin{tabular}{|c|c|c|c|c|c|c|c|}
\hline Anal ysis & $\begin{array}{c}\text { Sample } \\
\text { Identification } \\
\end{array}$ & $\begin{array}{l}\text { No. of } \\
\text { Samples } \\
\end{array}$ & Average & $\frac{(\mathrm{mg} / \mathrm{l})}{\text { Max im um }}$ & MinimLm & $\begin{array}{l}\text { Minimum } \\
\text { Criteria } \\
(\mathrm{mg} / \mathrm{l}) \\
\end{array}$ & $\begin{array}{c}\text { Detectable } \\
\text { Limit } \\
(\mathrm{mg} / \mathrm{l}) \\
\end{array}$ \\
\hline Hexavalent & PL & 2 & 0.03 & 0.04 & 0.02 & 1.0 & 0.015 \\
\hline Chromium $\left(\mathrm{Cr}_{\mathrm{r}}^{+6}\right)$ & $\begin{array}{l}\text { RP } \\
\text { PW } \\
\text { TW } \\
\text { STD } \\
\text { SLP } \\
\text { BLW }\end{array}$ & $\begin{array}{r}8 \\
32 \\
15 \\
9 \\
1 \\
9\end{array}$ & $\begin{array}{l}<0.028 \\
<0.024 \\
<0.02 \\
<0.08 \\
<0.015 \\
<0.022\end{array}$ & $\begin{array}{c}0.12 \\
0.11 \\
0.10 \\
0.48 \\
<0.015 \\
0.06\end{array}$ & $\begin{array}{l}<0.015 \\
<0.015 \\
<0.015 \\
<0.015 \\
<0.015 \\
<0.015\end{array}$ & $\begin{array}{c}1.0 \\
- \\
- \\
1.0 \\
1.0 \\
-\end{array}$ & $\begin{array}{l}0.015 \\
0.015 \\
0.015 \\
0.015 \\
0.015 \\
0.015\end{array}$ \\
\hline Copper (Cu) & $\begin{array}{l}\text { PL } \\
\text { RP } \\
\text { PW } \\
\text { TW } \\
\text { SFD } \\
\text { SLP } \\
\text { BUW }\end{array}$ & $\begin{array}{r}2 \\
8 \\
32 \\
15 \\
9 \\
1 \\
9\end{array}$ & $\begin{array}{c}<0.01 \\
<0.052 \\
<0.025 \\
<0.019 \\
<0.043 \\
0.08 \\
<0.016\end{array}$ & $\begin{array}{l}<0.01 \\
0.11 \\
0.16 \\
0.065 \\
0.092 \\
0.08 \\
0.037\end{array}$ & $\begin{array}{r}<0.01 \\
<0.01 \\
<0.01 \\
<0.01 \\
<0.01 \\
0.08 \\
<0.01\end{array}$ & $\begin{array}{c}0.2 \\
0.2 \\
- \\
- \\
0.2 \\
0.2 \\
-\end{array}$ & $\begin{array}{l}0.01 \\
0.01 \\
0.01 \\
0.01 \\
0.01 \\
0.01 \\
0.01\end{array}$ \\
\hline Cyanide $\left(\mathrm{CN}^{-1}\right)$ & $\begin{array}{l}\text { PL } \\
\text { RP } \\
\text { PW } \\
\text { IW } \\
\text { STD } \\
\text { SLP } \\
\text { BUW }\end{array}$ & $\begin{array}{r}2 \\
8 \\
33 \\
14 \\
9 \\
1 \\
9\end{array}$ & $\begin{array}{l}<0.02 \\
<0.02 \\
<0.02 \\
<0.02 \\
<0.03 \\
<0.02 \\
<0.02\end{array}$ & $\begin{array}{r}0.02 \\
0.02 \\
0.02 \\
0.02 \\
0.08 \\
<0.02 \\
0.02\end{array}$ & $\begin{array}{l}<0.02 \\
<0.02 \\
<0.02 \\
<0.02 \\
<0.02 \\
<0.02 \\
<0.02\end{array}$ & $\begin{array}{l}- \\
- \\
- \\
- \\
- \\
-\end{array}$ & $\begin{array}{l}0.02 \\
0.02 \\
0.02 \\
0.02 \\
0.02 \\
0.02 \\
0.02\end{array}$ \\
\hline Fluoride $\left(F^{-1}\right)$ & $\begin{array}{l}P L \\
\text { RP } \\
\text { PW } \\
\text { SLP } \\
\text { BUW }\end{array}$ & $\begin{array}{r}2 \\
8 \\
31 \\
1 \\
9\end{array}$ & $\begin{array}{l}0.9 \\
2.4 \\
1.7 \\
0.3 \\
2.6\end{array}$ & $\begin{array}{l}1.5 \\
2.8 \\
2.2 \\
0.3 \\
2.9\end{array}$ & $\begin{array}{l}0.2 \\
1.6 \\
1.1 \\
0.3 \\
2.1\end{array}$ & $\begin{array}{c}10 \\
10 \\
2.2 \\
10 \\
-\end{array}$ & $\begin{array}{l}0.2 \\
0.2 \\
0.2 \\
0.2 \\
0.2\end{array}$ \\
\hline Iron $(\mathrm{Fe})$ & $\begin{array}{l}\text { PL } \\
\text { RP } \\
\text { PW } \\
\text { TW } \\
\text { STD } \\
\text { SLP } \\
\text { BUW }\end{array}$ & $\begin{array}{r}2 \\
8 \\
32 \\
15 \\
9 \\
1 \\
8\end{array}$ & $\begin{array}{r}<0.02 \\
<0.07 \\
<0.02 \\
<0.05 \\
<0.56 \\
0.01 \\
<0.02\end{array}$ & $\begin{array}{l}0.02 \\
0.24 \\
0.03 \\
0.54 \\
1.69 \\
0.01 \\
0.02\end{array}$ & $\begin{array}{r}<0.01 \\
<0.01 \\
<0.01 \\
<0.01 \\
0.28 \\
0.01 \\
<0.01\end{array}$ & $\begin{array}{l}5 \\
5 \\
- \\
- \\
5 \\
5 \\
-\end{array}$ & $\begin{array}{l}0.01 \\
0.01 \\
0.01 \\
0.01 \\
0.01 \\
0.01 \\
0.01\end{array}$ \\
\hline Mercury $\left(\mathrm{Hg}_{\mathrm{g}}\right)$ & $\begin{array}{l}\text { PL } \\
\text { RP } \\
\text { PW } \\
\text { IW } \\
\text { STD } \\
\text { SLP } \\
\text { BUW }\end{array}$ & $\begin{array}{r}2 \\
8 \\
32 \\
14 \\
9 \\
1 \\
9\end{array}$ & $\begin{array}{r}<0.0002 \\
<0.0002 \\
<0.0002 \\
<0.0002 \\
<0.0002 \\
0.0001 \\
<0.0002\end{array}$ & $\begin{array}{l}0.0002 \\
0.0002 \\
0.0008 \\
0.0003 \\
0.0002 \\
0.0001 \\
0.0003\end{array}$ & $\begin{array}{r}<0.0001 \\
<0.0001 \\
<0.0001 \\
<0.0001 \\
<0.0001 \\
0.0001 \\
<0.0001\end{array}$ & $\begin{array}{c}- \\
- \\
0.002 \\
- \\
- \\
- \\
-\end{array}$ & $\begin{array}{l}0.0001 \\
0.0001 \\
0.0001 \\
0.0001 \\
0.0001 \\
0.0001 \\
0.0001\end{array}$ \\
\hline
\end{tabular}

\footnotetext{
$P L=$ Pantex Lake - (OW-WR-01)

$R P=$ Retention Playa - (OW-WR-08)

PW = Potable Water - (OW-WR-02, OW-WR-06, OW-WR-16, OW-WR-18, OW-WR-23, OW-WR-28)

$T W=$ Test Wells - (OW-WR-19, OW-WR-20)

STD = Sewage Treatment Discharge - (OW-WR-17)

SLP = Sanitary tandfill Playa (OW-WR-27)

BUW = Bushl and Experimental Station Water We11 (OW-WR-33)

Sample locations are identified on Fig. 6. and Page A-9.
} 
Table XXIII. CONTINUED

\begin{tabular}{|c|c|c|c|c|c|c|c|}
\hline Anal ysis & $\begin{array}{c}\text { Sample } \\
\text { Identification }\end{array}$ & $\begin{array}{l}\text { No. of } \\
\text { Samples }\end{array}$ & \multicolumn{3}{|c|}{$(\mathrm{mg} / \mathrm{l})$} & $\begin{array}{l}\text { Minimum } \\
\text { Criteria } \\
\text { (mg/l) }\end{array}$ & $\begin{array}{c}\text { Detectable } \\
\text { Limit } \\
\text { (mg/l) } \\
\end{array}$ \\
\hline \multirow{7}{*}{$\begin{array}{c}\text { Nitrate }\left(\mathrm{NO}_{3}{ }^{-1}\right) \\
\text { Reported as } \\
\mathrm{N} \text { (Nitrogen) }\end{array}$} & PL & 2 & 0.64 & 11.6 & 0.11 & - & 0.028 \\
\hline & RP & 8 & 2.0 & 6.4 & 0.13 & - & 0.028 \\
\hline & PW & 32 & 1.34 & 1.68 & 0.97 & 10.0 & 0.028 \\
\hline & $T W$ & 15 & $<1.67$ & 16.1 & $<0.028$ & - & 0.028 \\
\hline & STD & 9 & $<3.3$ & 10.1 & 0.78 & - & 0.028 \\
\hline & SLP & 1 & $<0.028$ & $<0.028$ & $<0.028$ & - & 0.028 \\
\hline & BUW & 9 & 1.31 & 1.56 & 1.03 & - & 0.028 \\
\hline \multirow[t]{7}{*}{ Lead $(\mathrm{Pb})$} & PL & 2 & $<0.001$ & $<0.001$ & $<0.001$ & 5.0 & 0.001 \\
\hline & RP & 8 & $<0.006$ & 0.029 & $<0.001$ & 5.0 & 0.001 \\
\hline & PW & 32 & $<0.002$ & 0.004 & $<0.001$ & 0.05 & 0.001 \\
\hline & $T W$ & 15 & $<0.002$ & 0.006 & $<0.001$ & - & 0.001 \\
\hline & STD & 9 & $<0.002$ & 0.005 & $<0.001$ & 5.0 & 0.001 \\
\hline & SLP & 1 & $<0.001$ & $<0.001$ & $<0.001$ & 5.0 & 0.001 \\
\hline & BUW & 9 & $<0.002$ & 0.005 & $<0.001$ & - & 0.001 \\
\hline \multirow[t]{7}{*}{ Phenols } & PL & 2 & $<0.012$ & 0.012 & $<0.012$ & - & 0.012 \\
\hline & $\mathrm{RP}$ & 8 & $<0.012$ & 0.012 & $<0.012$ & - & 0.012 \\
\hline & PW & 30 & $<0.012$ & $<0.012$ & $<0.012$ & - & 0.012 \\
\hline & $T W$ & 15 & $<0.017$ & 0.037 & $<0.012$ & - & 0.012 \\
\hline & STD & 9 & $<0.012$ & $<0.012$ & $<0.012$ & - & 0.012 \\
\hline & SLP & 1 & 0.012 & 0.012 & 0.012 & - & 0.012 \\
\hline & BUW & 9 & $<0.012$ & $<0.012$ & $<0.012$ & - & 0.012 \\
\hline \multirow[t]{7}{*}{ Phosphate $\left(\mathrm{PO}_{4}^{-3}\right)$} & $P L$ & 2 & 0.30 & 0.54 & 0.05 & - & 0.036 \\
\hline & $\mathrm{RP}$ & 8 & 0.91 & 1.49 & 0.38 & - & 0.036 \\
\hline & PW & 31 & $<0.076$ & 0.29 & $<0.036$ & - & 0.036 \\
\hline & $T W$ & 15 & $<0.06$ & 0,15 & $<0.036$ & - & 0.036 \\
\hline & STD & 9 & 0.79 & 1.28 & 0.54 & - & 0.036 \\
\hline & SLP & 1 & $<0.036$ & $<0.036$ & $<0.036$ & - & 0.036 \\
\hline & BUW & 9 & 0.058 & 0.10 & $<0.036$ & - & 0.036 \\
\hline \multirow[t]{6}{*}{ Sulfate $\left(\mathrm{SO}_{4}{ }^{-2}\right)$} & $\mathrm{PL}$ & 2 & 34 & 48 & 19 & 200 & 1 \\
\hline & $\mathrm{RP}$ & 8 & 119 & 298 & 61 & 200 & 1 \\
\hline & PW & 31 & 17 & 39 & 8 & - & 1 \\
\hline & $T W$ & 15 & 28 & 39 & 13 & - & 1 \\
\hline & STD & 9 & 93 & 182 & 48 & 200 & 1 \\
\hline & BUW & 9 & 33 & 35 & 31 & - & 1 \\
\hline \multicolumn{3}{|c|}{$P L=P a n t e x$ Lake $-(O W-W R-01)$} & & & & & \\
\hline \multicolumn{8}{|c|}{$R P=$ Retention Playa $-(O W-W R-O B)$} \\
\hline \multicolumn{8}{|c|}{$P W=$ Potable Water $-($ OW-WR-02, OW-WR-06, OW-WR-16, OW-WR-18, OW-WR-23, OW-WR-28) } \\
\hline \multicolumn{8}{|c|}{$I W=$ Test Wells $-($ OW-WR-19, OW-WR-20) } \\
\hline \multicolumn{8}{|c|}{ STD $=$ Sewage Treatment Discharge $-(O W-W R-17)$} \\
\hline \multicolumn{8}{|c|}{ SLP = Sanitary Landfill Playa (OW-WR-27) } \\
\hline BUW $=$ Bushl and $E_{x p}$ & perimental Stati & nater & $1(\mathrm{OW}-\mathrm{W})$ & 33) & & & \\
\hline
\end{tabular}


Table XXIII. CONTINUED

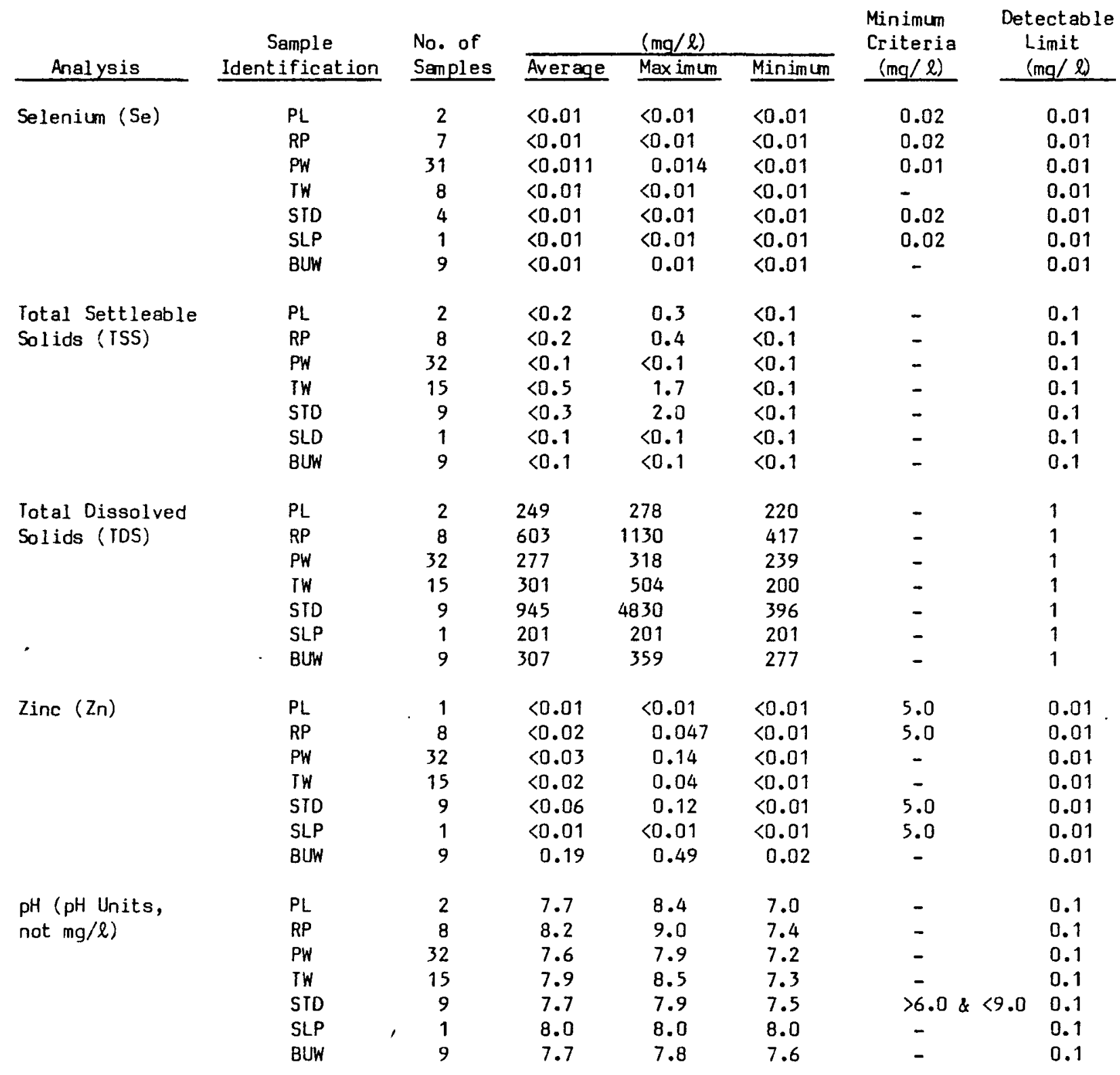

\footnotetext{
$P L=$ Pantex Lake - (OW-WR-01)

RP $=$ Retention Playa - (OW-WR-08)

PW = Potable Water - (OW-WR-02, OW-WR-06, OW-WR-16, OW-WR-18, OW-WR-23, OW-WR-28)

$T W=$ Test Wells - (OW-WR-19, OW-WR-20)

STD = Sewage Treatment Discharge - (OW-WR-17)

SLP = Sanitary Landfill Playa (OW-WR-27)

BUW = Bushland Experimental Station Water Well (OW-WR-33)

Sample locations are identified on Fig. 6. and Page A-9.
} 
Table XXIII. CONTINUED

\begin{tabular}{|c|c|c|c|c|c|c|c|}
\hline Analysis & $\begin{array}{c}\text { Sample } \\
\text { Identification } \\
\end{array}$ & $\begin{array}{l}\text { No. of } \\
\text { Samples } \\
\end{array}$ & Average & $\frac{(\mathrm{mg} / \mathrm{l})}{\text { Max imum }}$ & Minim um & $\begin{array}{l}\text { Minimum } \\
\text { Criteria } \\
\text { (mg/l) } \\
\end{array}$ & $\begin{array}{c}\text { Detectable } \\
\text { Limit } \\
(\mathrm{mg} / \mathrm{l}) \\
\end{array}$ \\
\hline \multirow[t]{7}{*}{$0 i l / G r e a s e$} & $P L$ & 2 & $<3$ & $<3$ & $<3$ & - & 3 \\
\hline & $\mathrm{RP}$ & 8 & $<3$ & $<3$ & $<3$ & - & 3 \\
\hline & PW & 32 & $<3$ & $<3$ & $<3$ & - & 3 \\
\hline & $T W$ & 15 & $<3$ & $<3$ & $<3$ & - & 3 \\
\hline & STD & 9 & $<3.2$ & 3.6 & $<3$ & - & 3 \\
\hline & SLP & 1 & $<3$ & $<3$ & $<3$ & - & 3 \\
\hline & BUW & 9 & $<3$ & $<3$ & $<3$ & - & \\
\hline \multirow[t]{7}{*}{ Hardness } & $P L$ & 2 & 384 & 570 & 197 & - & 1 \\
\hline & $\mathrm{RP}$ & 8 & 229 & 278 & 146 & - & 1 \\
\hline & $\mathrm{PW}$ & 32 & 198 & 234 & 120 & - & 1 \\
\hline & $T W$ & 15 & 151 & 231 & 105 & - & 1 \\
\hline & STD & 9 & 240 & 285 & 140 & - & 1 \\
\hline & SLP & 1 & 111 & 111 & 111 & - & 1 \\
\hline & BUW & 9 & 197 & 299 & 89 & - & 1 \\
\hline \multirow[t]{7}{*}{ RDX } & $P L$ & 2 & $<0.07$ & $<0.07$ & $<0.07$ & - & 0.07 \\
\hline & $\mathrm{RP}$ & 8 & $<0.07$ & $<0.07$ & $<0.07$ & - & 0.07 \\
\hline & PW & 32 & $<0.07$ & $<0.07$ & $<0.07$ & - & 0.07 \\
\hline & $T W$ & 15 & $<0.07$ & $<0.07$ & $<0.07$ & - & 0.07 \\
\hline & STD & 9 & $<0.07$ & $<0.07$ & $<0.07$ & - & 0.07 \\
\hline & SLP & 1 & $<0.07$ & $<0.07$ & $<0.07$ & - & 0.07 \\
\hline & BUW & 9 & $<0.07$ & $<0.07$ & $<0.07$ & - & 0.07 \\
\hline \multirow[t]{7}{*}{ HMX } & PL & 2 & $<0.11$ & $<0.11$ & $<0.11$ & - & 0.11 \\
\hline & $R P$ & 8 & $<0.11$ & $<0.11$ & $<0.11$ & - & 0.11 \\
\hline & PW & 32 & $<0.12$ & 0.12 & $<0.11$ & - & 0.11 \\
\hline & $T W$ & 15 & $<0.11$ & $<0.11$ & $<0.11$ & - & 0.11 \\
\hline & STD & 9 & $<0.12$ & 0.12 & $<0.11$ & - & 0.11 \\
\hline & SLP & 1 & $<0.11$ & $<0.11$ & $<0.11$ & - & 0.11 \\
\hline & BUW & 9 & $<0.11$ & $<0.11$ & $<0.11$ & - & 0.11 \\
\hline \multirow[t]{7}{*}{ PEIN } & $P L$ & 1 & $<0.25$ & $<0.25$ & $<0.25$ & - & 0.25 \\
\hline & $\mathrm{RP}$ & 6 & $<0.25$ & $<0.25$ & $<0.25$ & - & 0.25 \\
\hline & PW & 32 & $<0.25$ & $<0.25$ & $<0.25$ & - & 0.25 \\
\hline & $T W$ & 15 & $<0.25$ & $<0.25$ & $<0.25$ & - & 0.25 \\
\hline & STD & 9 & $<0.25$ & $<0.25$ & $<0.25$ & - & 0.25 \\
\hline & SLP & 1 & $<0.25$ & $<0.25$ & $<0.25$ & - & 0.25 \\
\hline & BUW & 9 & $<0.25$ & $<0.25$ & $<0.25$ & - & 0.25 \\
\hline
\end{tabular}

\footnotetext{
$P L=$ Pantex Lake - (OW-WR-01)

$R P=$ Retention Playa - (OW-WR-OB)

PW = Potable Water - (OW-WR-02, OW-WR-06, OW-WR-16, OW-WR-18, OW-WR-23, OW-WR-28)

$T W=$ Test Wells - (OW-WR-19, OW-WR-20)

STD = Sewage Treatment Discharge - (OW-WR-17)

SLP = Sanitary Landfil1 Playa (OW-WR-27)

BUW = Bushland Experimental Station Water Wel1 (OW-WR-33)

Sample locations are ident ified on Fig. 6. and Page A-9.
} 
Table XXIII. CONTINUED

\begin{tabular}{|c|c|c|c|c|c|c|c|}
\hline Analys is & $\begin{array}{c}\text { Sample } \\
\text { Identification }\end{array}$ & $\begin{array}{l}\text { No. of } \\
\text { Samples }\end{array}$ & Average & $\frac{(\mathrm{mg} / \mathrm{l})}{\operatorname{Max} \mathrm{im} u m}$ & Minimum & $\begin{array}{l}\text { Minimum } \\
\text { Criteria } \\
\text { (mg/l) } \\
\end{array}$ & $\begin{array}{c}\text { Detectable } \\
\text { Limit } \\
(\mathrm{mg} / \mathrm{l}) \\
\end{array}$ \\
\hline \multirow{4}{*}{ Endrin } & $\mathrm{PL}$ & 2 & $<0.0002$ & $<0.0002$ & $<0.0002$ & - & 0.0002 \\
\hline & $\mathrm{RP}$ & 7 & $<0.0003$ & 0.0007 & $<0.0002$ & - & 0.0002 \\
\hline & PW & 28 & $<0.0003$ & 0.0006 & $<0.0002$ & 0.0002 & 0.0002 \\
\hline & BUW & 8 & $<0.0003$ & 0.0006 & $<0.0002$ & - & 0.0002 \\
\hline \multirow[t]{4}{*}{ Lindane } & PL & 2 & $<0.0004$ & $<0.0004$ & $<0.0004$ & - & 0.0004 \\
\hline & $\mathrm{RP}$ & 8 & $<0.005$ & 0.005 & $<0.0004$ & - & 0.0004 \\
\hline & PW & 32 & $<0.0009$ & 0.0039 & $<0.0004$ & 0.004 & 0.0004 \\
\hline & BuW & 9 & $<0.0058$ & 0.0058 & $<0.0004$ & - & 0.0004 \\
\hline \multirow[t]{4}{*}{ Methoxychlor } & PL & 2 & $<0.1$ & $<0.1$ & $<0.1$ & - & 0.1 \\
\hline & $\mathrm{RP}$ & 8 & $<0.1$ & $<0.1$ & $<0.1$ & - & 0.1 \\
\hline & PW & 32 & $<0.1$ & $<0.1$ & $<0.1$ & 0.1 & 0.1 \\
\hline & BUW & 9 & $<0.1$ & $<0.1$ & $<0.1$ & - & 0.1 \\
\hline \multirow{4}{*}{$2,4-D$} & PL & 2 & $<0.1$ & $<0.1$ & $<0.1$ & - & 0.1 \\
\hline & $\mathrm{RP}$ & 8 & $<0.1$ & $<0.1$ & $<0.1$ & - & 0.1 \\
\hline & $P W$ & 32 & $<0.1$ & $<0.1$ & $<0.1$ & 0.1 & 0.1 \\
\hline & BUW & 9 & $<0.1$ & $<0.1$ & $<0.1$ & - & 0.1 \\
\hline \multirow[t]{4}{*}{$2,4,5-T$} & $\mathrm{PL}$ & 2 & $<0.01$ & $<0.01$ & $<0.01$ & - & 0.01 \\
\hline & $R P$ & 8 & $<0.01$ & $<0.01$ & $<0.01$ & - & 0.01 \\
\hline & $P W$ & 32 & $<0.01$ & $<0.01$ & $<0.01$ & 0.01 & 0.01 \\
\hline & BUW & 9 & $<0.01$ & $<0.01$ & $<0.01$ & - & 0.01 \\
\hline$B O D$ (5-day) & SID & 25 & 27.9 & 55.9 & 6.6 & $\begin{array}{l}\text { 50, 30-day } \\
\text { avg. } 150, \\
\text { grab }\end{array}$ & $y$ \\
\hline $\mathrm{COD}$ & $\mathrm{RP}$ & 40 & 52.1 & 95 & $<50$ & 300 , grab & so \\
\hline $\begin{array}{l}\text { Fecal Coliform } \\
(\text { colonies } / 100 \mathrm{ml})\end{array}$ & PW & 50 & 0.0 & 5 & 0 & 1 & 1 \\
\hline
\end{tabular}

${ }^{a}$ Varies with dilution

$\mathrm{b}_{\text {See Reference } 23 .}$

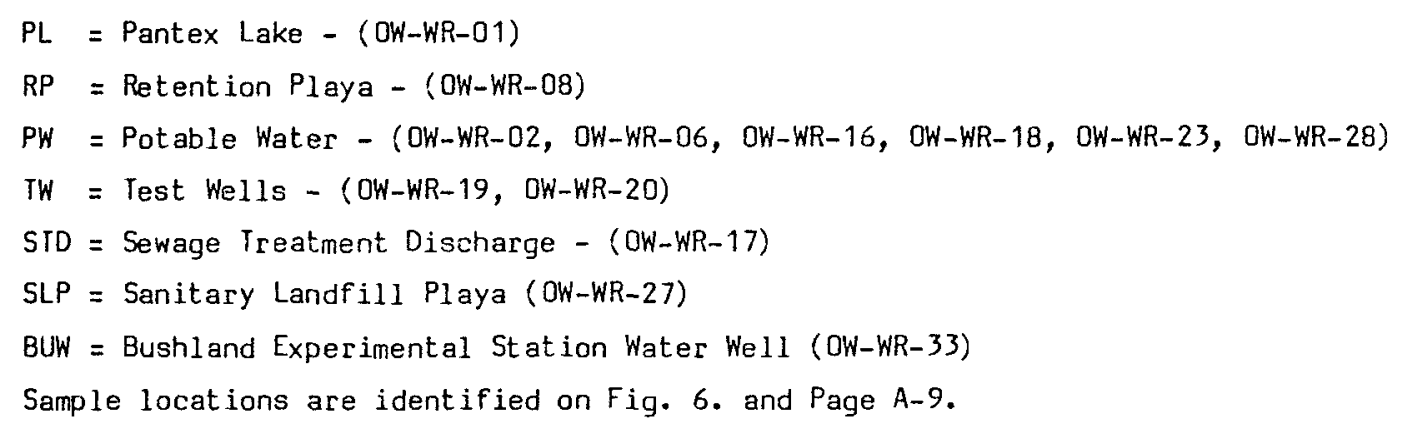




\section{REFERENCES}

1. U.S. Department of Energy, DOE Explosives Safety Manual, DOE/EV/06194-1, June 1982 .

2. U.S. Army, DARCOM Regulation Safety Manual, DARCOM 385-100, August 1981.

3. Letter Cecil L. Bradford, Texas Air Control Board to P. R. Wagner, United States Energy Research and Development Administration, dated July 9, 1976.

4. Energy and Air Quality, U.S. Department of Energy, Report Number DOE/EV/10154-3, December 1981.

5. McDonald, James R., "Assessment of Tornado and Straight Wind Risks at the Pantex, Texas Site," McDonald, Menta and Minor Consulting Engineers, December 1979 .

6. Seismic Hazard Analysis, Pantex Ordnance Plant, Amarillo, Texas, TERA Corporation, Berkeley, CA, May, 1980 (Draft).

7. Uniform Building Code, 1982 .

8. Percolation of Water Through Pullman Soils, Texas High Plains, The Texas Agricultural Experimental Station, 1971.

9. Basin Recharge of the Ogallala Aquifer, Southwestern Great Plains Research Center, 1972 .

10. Hydrogeology of a Playa Near Amarillo, Texas, Southwestern Great Plains Research Center, 1971.

11. 1980 Census of Population and Housing, U.S. Department of Commerce, Report No. PHC80-V-45, March 1981.

12. Users Guide for the Climatalogical Dispersion Model by Adrain D. Busse and John R. Zimmerman, EPA-R4-73-024, Decémber 1973.

13. Draft Environmental Impact Statement, Pantex Plant Site, Amarillo, Texas, U.S. Department of Energy, Report Number DOE/EIS-0098-D, December 1982 .

14. Local Climatological Data, U.S. Department of Commerce, National Oceanic and Atmospheric Administration, January 1982 through December 1982 .

15. Martin, J. A., Jr., "Calculations of Environmental Radiation Exposures and Population Doses Due to Effluents from a Nuclear Fuel Reprocessing Plant," Radiation Data and Reports, Vol. 15, No. 2 February 1973.

16. DOE Order 5480.1, Chapter XI, Requirements for Radiation Protection.

17. National Interim Primary Drinking Water Regulations, Title 40, Part 141, Environmental Protection Agency, December 24, 1975. 
18. General Regulations Incorporated Into Permits, Chapter XIX, 156.19.15.002, Texas Water Development Board, Austin, Texas.

19. General Regulations Incorporated Into Permits, Chapter XIX, Hazardous Metals, 156.19.15.001 through 156.19.15.009, Texas Water Development Board, Austin, Texas.

20. Standard Methods for the Examination of Water and Waste Water, Fifteenth Edition, American Public Health Association, New York, NY, 1980.

21. Methods for Chemical Analyses of Water and Wastes, Environmental Protection Agency, Water Quality Office, Analytical Control Laboratory, Cincinnati, Ohio, $1971,16020,07 / 71$.

22. Analysis of Pesticide Residues in Human and Environmental Samples, Environmental Protection Agency, Toxicology Division, Research Triangle Park, NC, 27711, June 1977.

23. G. W. Dawson, "The Chemical Toxicity of Elements," Pacific Northwest Laboratories, Report Number BNWL-1815, June 1974. 


\section{STANDARDS FOR RADIOACTIVITY IN AIR AND WATER}

Generally standards for radioactivity in air and water are derived from DOE Order 5480.1A and its Attachment XI-1(16). The Radioactivity Concentration Guides (RCG's) for uncontrolled areas are generally the concentrations at which an individual, if continually exposed, would receive about 0.500 rem total body dose equivalent per year. RCG's for the general population are approximately $1 / 3$ of the RCG's for uncontrolled areas so that the general population will receive no more than $0.170 \mathrm{rem}$ total dose equivalent per year or not more than $0.500 \mathrm{rem}$ to the lungs or kidney when these serve as the critical organ.

In the case of mixtures of specific nuclides, the resultant RCG is determined such that the sum of the ratios of the concentrations of each nuclide to its respective RCG does not exceed unity. Nuclide concentrations are not considered part of a mixture whenever the concentration to RCG ratio is less than or equal to 0.1 and the sum of the ratios for all nuclides is less than 0.25 .

\section{RADIOACTIVITY CONCENTRATION GUIDES FOR SOIL AND VEGETATION}

Presently there are no published generally accepted RCG's for radionuclides in soil and vegetation.

\section{NON-RADIOACTIVE WATER QUALITY STANDARDS}

Non-radioactive analyses are compared to applicable EPA Primary Drinking Water Standards 40 CFR 141, EPA Secondary Treatment Regulations $40 \mathrm{CFR} 143$, and Texas Water Development Board Regulations, Chapter XIX(17, 18, 19).

\section{STATISTICAL METHODS}

Nonradioactive measurements are based on analog representations of physical parameters and a lower limit exists as to the sensitivity of the techniques and instrumentation employed. When measurements of non-radioactive parameters were performed and no indication in excess of lower detection limit was observed, the result is presented as less than $(<)$ the minimum detectable limit. Average values were calculated by assuming the minimum detectable limit where positive indications were not obtained and portrayals of the final average as a "less than" value. The "less than" value for an average therefore indicates the assumption of the minimum detectable limit for one or more terms within the average.

Radioactive measurements, however, are subject to the statistical nature of radioactive decay and are presented in conjunction with an associated statistical error. All errors presented represent counting errors only; no attempt has been made to quantify systematic errors. The minimum detectable limits of the radioactive parameters were calculated based on USiNRC Regulatory Guide 4.14. Negative numbers can arise from statistical fluctuations in the radioactive decay of the sample, the blank, or in the background of the counting device. Averages which are less than zero in value (i.e., negative) are presented as being less than the minimum detectable limit.

$$
A-1
$$


WATER

\section{ANALYTICAL METHODS (NON-RADIOACTIVE)}

In general, non-radioactive analyses of water were performed in accordance with standard methods $(20,21)$. High explosives were analyzed by HPLC (High Performance Liquid Chromatography) utilizing a UV absorption detector. Pesticides were analyzed by vapor phase gas chromatography(22).

\section{ANALYTICAL METHODS (RADIOACTIVE)}

\section{AIR}

\section{AIR FILTERS}

Gross Alpha, Gross Beta

Fil ter paper samples are counted for Gross Alpha and Gross Beta radioactivity on a low background internal flow proportional counter which simultaneously counts alpha and beta activity. Appropriate corrections are applied for absorption and counter efficiency.

\section{Total Uranium}

The sample is digested to dryness with strong acids and re-dissolved in $5 \circ \mathrm{HNO}_{3}$. The Uranium is separated by co-precipitation with $\mathrm{AlPO}_{4}$. Then the precipitate is dissolved in $2 \mathrm{~N} \mathrm{Al}\left(\mathrm{NO}_{3}\right)_{3}$ and extracted into ethyl acetate. The ethyl acetate is dried and the sample transferred to a stainless steel planchet for counting in an internal flow proportional counter for its alpha activity.

\section{Plutonium-239}

A $\mathrm{Pu}-236$ tracer is added to the filter paper sample, totally dissolved, and converted to the nitrate by additions of $\mathrm{HNO}_{3}$. The Plutonium is separated using an ion exchange resin, eluted off the column, and electroplated on a stainless steel disc. A solid state alpha spectrometer is utilized to count the disc, and chemical recovery is determined from the tracer peak.

\section{SILICA GEL}

Moisture is removed from the silica gel using a distillation apparatus. A portion of the distillate is mixed with a scintillation solution and counted in a liquid scintillation spectrometer. The atmospheric tritium concentration is related to the tritium concentration of the distillate through the average absolute humidity for the month during which the sample was collected. 


\section{Gross Alpha (Dissolved Solids)}

After thorough agitation, a suitable aliquot is taken for analysis. The aliquot is acidified and evaporated to dryness on a hot plate after which the residue is dissolved in $1 \mathrm{~N}^{-\mathrm{HNO}_{3}}$ and transferred to a tared planchet. Gross Alpha radioactivity is then determined by counting the planchet in an internal flow propor tional counter (Canberra Model 2201).

The result is corrected for counter efficiency and self-absorption if an appreciable solid residue is obtained. For thickness greater than $1 \mathrm{mg} / \mathrm{cm}^{2}$, a correction must be applied.

\section{Gross Beta (Dissolved Solids)}

This same method is used as described under Gross Alpha (Dissolved Solids). A self-absorption correction factor is applied when the sample weight exceeds $1 \mathrm{mg} / \mathrm{cm}^{2}$.

\section{Gross Alpha (Suspended Solids)}

A suitable aliquot of water is filtered through a Millipore filter $(0.45 \mu \mathrm{m})$. The filter is counted utilizing a Canberra Model 2201 instrument. Corrections are applied for self-absorption.

Gross Beta (Suspended Solids)

The same method is used as described under Gross Alpha (Suspended Solids).

\section{Total Uranium}

The sample is digested to dryness with strong acids and re-dissolved in 5\% $\mathrm{HNO}_{3}$. The Uranium is separated by co-precipitation with $\mathrm{AlPO}_{4}{ }^{\circ}$. Then the precipitate is dissolved in $2 \mathrm{~N} \mathrm{Al}\left(\mathrm{NO}_{3}\right)_{3}$ and extracted into ethyl acetate. The ethyl acetate is dried and the sample transferred to a stainless steel planchet for counting in an internal flow proportional counter for its alpha activity.

\section{$\underline{\text { plutonium-239 }}$}

The total water sample is aliquoted and acidified. A Pu-236 tracer is added to the aliquot and brought to dryness. The residue is ashed and dissolved in nitric acid. Plutonium is separated using an ion exchange resin, eluted of $\mathrm{f}$ the column, and electroplated on a stainless steel disc. A solid state alpha spectrometer is utilized to count the disc and chemical recovery is determined from the tracer peak. 
$\underline{\text { Tritium }}$

The water sample is distilled to remove quenching materials and non-volatile radioactive materials. Distillation is carried to dryness to ensure complete transfer of the tritium to the distillate. A portion of the distillate is mixed with scintillation solution and counted in a liquid scintillation spectrometer. Standard tritium and background samples are prepared and counted alternately to nullify errors produced by aging of the scintillation medium or instrument drif $t$.

\section{$\underline{\text { Radium }-226}$}

Radium in the water sample is co-precipitated with barium sulfate. The bariumradium sulfate is separated from other alpha-emitters by dissolution with disodium EDTA, then reprecipitated as the sulfate, by addition of glacial acetic acid. The barium-radium sulfate is then transferred to a planchet for gross alpha counting.

\section{$\underline{\text { Radium-228 }}$}

The radium in the water sample is collected by coprecipitation with barium and lead sulfate, and purified by reprecipitation from EDTA solution. After a 36-hour ingrowth of actinium-228 from radium -228 , the actinium -228 is carried on yttrium oxalate, purified and beta counted.

\section{SOIL}

\section{Total Uranium}

The sample is digested to dryness with strong acids and re-dissolved in $5 \% \mathrm{HNO}_{3}$. The Uranium is separated by co-precipitation with $\mathrm{AlPO}_{4^{*}}$. Then the precipitate is dissolved in $2 \mathrm{~N} \mathrm{Al}\left(\mathrm{NO}_{3}\right)_{3}$ and extracted into ethyl acetate. The ethyl acetate is dried and the sample transferred to a stainless steel planchet for counting in an internal flow porportional counter for its alpha activity.

\section{Plutonium}

The soil sample is totally dissolved using a $40 \%$ solution of $\mathrm{HF}$ and a $\mathrm{Pu}-236$ tracer is added before dissolution. After fuming with HF, the sample is brought up with $\mathrm{HNO}_{3}$. An ion exchange column is utilized to separate the Plutonium which is subsequently eluted off the column and electroplated on a stainless steel counting disc. This disc is counted on a solid state alpha spectrometer and recovery is determined from the tracer peak.

\section{VEGETATION}

$\underline{\text { Total Uranium }}$

Initially, the dry vegetation is ashed in a muffle furnace. The aliquot is digested to dryness with strong acids and re-dissolved in 5\% $\mathrm{HNO}_{3}$. The Uranium is 
separated by co-precipitation with $\mathrm{AlPO}_{4}$. Then the precipitate is dissolved in $2 \mathrm{~N} \mathrm{Al}\left(\mathrm{NO}_{3}\right)_{3}$ and extracted into ethyl acetate. The ethyl acetate is dried and the sample transferred to a stainless steel planchet for counting in an internal flow proportional counter for its alpha activity.

\section{$\underline{\text { Tritium }}$}

The water is extracted from the vegetation sample by azeotropic distillation with benzene. The distillation is carried to dryness to ensure complete transfer of the tritium. A portion of the distillate is mixed with scintillation solution and counted utilizing a liquid scintillation spectrometer. Standard tritium and background samples are prepared and counted alternately to nullify errors produced by aging of the scintillation medium or instrument drift.

\section{QUALITY CONTROL}

\section{$\underline{\text { Radioactive }}$}

During 1983 radioactive analyses were performed under contract by Camp Dresser and McKee, Inc. (CDM) of Wheat Ridge (Denver), Colorado. The QA program in the CDM Radiochemistry Laboratory is designed to comply with the requirements of Title 10 CFR 50, Appendix B, and NRC specifications in Regulatory Guide 4.15.

The following summary of the CDM QA Program is designed to follow the NRC Regulatory Guide 4.15 , Parts 6.1 thru 6.4.

Part 6.1 Radionuclide Reference Standards Used for Efficiency and Consistency Checks, and 6.2 Background Counting Rate Checks.

Liquid Scintillation system efficiency and consistency is maintained using known $\mathrm{C}-14$ and $\mathrm{H}-3$ standard solutions from Packard Instrument Company and a known $\mathrm{H}-3$ solution from EPA. The EPA standard is run with each sample set to ensure acceptability of the entire set of results. Blanks are determined using a standard background vial supplied by Packard Instrument Company. The vial is run thrice for a duration of ten minutes each with each sample set. Log books and control charts are maintained for each instrument.

Alpha Spectometry system efficiency and consistency is maintained by weekly verification checks, using a natural thorium source (traceable to NBS) electroplated onto a clean stainless steel disc. Blanks are determined using clean stainless steel discs. EPA standard solutions are counted with each sample set (10 to 20 samples), and charts are maintained to ensure data acceptability.

Internal Flow Proportional Counter (for alpha activity) efficiency and consistency is assured through a daily check with a Pu-239 source and a background count. Additionally, gross alpha absorption curves are prepared to monitor instrument efficiency and consistency for differing amounts of sample residue, and EPA standard solutions containing known amounts of radium-226, thorium-230, or polonium-210 are counted for each corresponding 
sample set ( 10 to 20 samples) run. Logs and control charts are maintained on these standards.

\section{Part 6.3 Quality Control Samples}

Reference standards were covered in the preceding section. An average of $10 \%$ of the sample load is run in duplicate. Reagent blanks (samples made up entirely of reagents in the same proportions they would be used for each type of analysis performed) are run with every set of samples.

CDM's Radiochemistry Lab participates in the EPA's Environmental Radioactivity Laboratory Intercomparison Studies Program. Samples are submitted monthly by the EPA for either radium-226, radium-228, total uranium, or gross alpha and gross beta, plutonium-239, or tritium analysis. The Lab is certified by the EPA's Environmental Radioactivity Laboratory Intercomparison Studies Program under the Safe Drinking Water Act.

\section{Part 6.4 Computational Checks}

For calculation verification, CDM has a three phase system set up. In phase one, the calculations are performed and approved by the analyst. Phase two is a complete check of all the original calculations by a second technician who is certified to do the specific analysis. Phase three involves a third check on $\dot{a}$ fraction of the calculations by the superviscr, who then gives final approval.

\section{Additional QA Procedures}

CDM's Quality Assurance Program is overseen by a Quality Assurance Manager whose responsibility is to carry out periodic technical audits, to oversee inter- and intra-laboratory analyses, to prepare quality assurance related documents, to oversee the personnel certification program and other Quality Assurance related activities. The Quality Assurance Manager reports directly to the Director of Analytical Services.

\section{Non-Radioactive}

During 1983 non-radioactive water analyses, except BOD, COD, and fecal coliform, were performed under contract by Camp Dresser and McKee, Inc. (CDM). The QA program in the CDM Water Laboratory is, like the Radiochemistry Lab, designed to conform with the requirements of NRC Regulatory Guide 4.15. Reference materials procured from EPA or NBS are run with each sample series. Control charts are maintained on these standards. Duplicates and spikes are used to ensure integrity of results.

The Laboratory participates in Intercomparison Programs sponsored by EPA, the Departments of Health of the states of Arizona and Colorado, and the state of Oklahoma. The Laboratory is certified by the states of Colorado, Montana, New Mexico and Oklahoma for water analysis, per the Safe Drinking Water Act (Texas does not certify labs). Computational checks, personnel training and other QA procedures are identical to procedures in the Radiochemistry Laboratory. 
Fecal Coliform samples were analyzed by the Amarillo Bi-City County Health Department of the City of Amarillo. This is a state-supported state-certified laboratory.

BOD and COD were run internally, using procedures in Standard Methods, 15th Edition. An Alpha-Trol standard was run with each set of samples.

\section{RADIOACTIVITY CONCENTRATION GUIDES (RCG's)(16)}

\section{General Population}

\begin{tabular}{|c|c|c|c|}
\hline & $\begin{array}{c}\text { Air } \\
(\mu \mathrm{Ci} / \mathrm{m} \ell) \\
\end{array}$ & $\begin{array}{r}W \\
(\mu \\
\end{array}$ & $\begin{array}{l}\text { ter } \\
i / m \ell)\end{array}$ \\
\hline Plutonium- 239 & $2 \times 10-14$ & 2 & $10^{-6}$ \\
\hline Tritium & $7 \times 10-8$ & $1:$ & $10^{-3}$ \\
\hline Uranium-238 & $1 \times 10-12$ & 2 & $10^{-7}$ \\
\hline Radium- 226 & $1 \times 10-12$ & 1 & $10^{-8}$ \\
\hline Radi um- 228 & $7 \times 10-13$ & 1 & $10^{-8}$ \\
\hline
\end{tabular}

PERMISSIBLE ANNUAL POPULATION DOSES FOR KIDNEY AND WHOLE BODY EXPOSURES (16)

Reference Point
Site boundary and highest
exposed individual or
group

General population

\begin{tabular}{ll}
$\begin{array}{l}\text { Kidney } \\
\text { (rem) }\end{array}$ & Lung \\
& $\underline{\text { rem })}$ \\
\hline
\end{tabular}

$\begin{array}{ll}1.50 & 1.50 \\ 0.500 & 0.500\end{array}$

0.500

0.170 
WATER QUALITY CRITERIA

\begin{tabular}{|c|c|c|c|c|}
\hline & $\begin{array}{c}\text { EPA } \\
\text { Dr inking } \\
\text { Water } \\
\text { Regul ations } \\
(\mathrm{mg} / \mathrm{l}) \\
\end{array}$ & $\begin{array}{c}\text { Desired Maximum } \\
\text { Levels of El ements } \\
\text { for Irrigation Waters(23) } \\
(\mathrm{mg} / \mathrm{l}) \\
\end{array}$ & $\begin{array}{c}\text { Texas Water } \\
\text { Development Board } \\
\text { Water Quality } \\
\text { Levels - Inl and } \\
\text { Waters } \\
(\mathrm{mg} / \mathrm{l}) \\
\end{array}$ & $\begin{array}{l}\quad \text { Texas Water } \\
\text { Commission, Permit } \\
\text { To Dispose of Waste } \\
\text { Permit No. } 02296 \\
\end{array}$ \\
\hline Arsenic (As) & 0.05 & 0.1 & 0.1 & \\
\hline Barium（Ba） & 1.0 & 1.0 & 1.0 & \\
\hline Cadmi um (Cd) & 0.010 & 0.005 & 0.05 & \\
\hline Chromium (Total) & 0.05 & - & 0.5 & \\
\hline Chromium $(+6)$ & - & 1.0 & - & \\
\hline Copper & - & 0.2 & 0.5 & \\
\hline Cyanide & - & - & - & \\
\hline Fluoride & $2.2^{*}$ & 10 & - & \\
\hline Iron & - & 5.0 & - & \\
\hline Lead & 0.05 & 5.0 & 0.5 & \\
\hline Mercury & 0.002 & - & 0.005 & \\
\hline Nitrate (as N) & 10 & - & - & \\
\hline Phenol & - & - & - & \\
\hline Phosphate & - & - & - & \\
\hline Silver & 0.05 & - & 0.05 & \\
\hline Sulfate & - & 200 & - & \\
\hline Selenium & 0.01 & 0.02 & 0.05 & \\
\hline $\begin{array}{l}\text { Total Dissolved } \\
\text { Solids }\end{array}$ & - & - & - & \\
\hline Suspended Solids & - & - & - & \\
\hline Zinc & - & 5.0 & 1.0 & \\
\hline BOD (5 day) & - & - & - & $\begin{array}{l}\text { 50, 30-day Avg. } \\
150 \text {, Grab }\end{array}$ \\
\hline Fecal Coliform & $1 \mathrm{ct} / 100 \mathrm{ml}$ & - & - & \\
\hline $\begin{array}{l}\mathrm{pH} \\
\mathrm{COD}\end{array}$ & - & - & - & $\begin{array}{l}>6.0 \&<9.0 \\
300, \text { Grab }\end{array}$ \\
\hline \multicolumn{5}{|l|}{ PESTICIDES } \\
\hline Endrin & 0.0002 & - & - & \\
\hline Lindane & 0.004 & - & - & \\
\hline Methoxychlor & 0.1 & - & - & \\
\hline Toxaphene & 0.005 & - & - & \\
\hline $2,4-D$ & 0.1 & - & - & \\
\hline $2,4,5-T P$ (Silvex) & 0.01 & - & - & \\
\hline
\end{tabular}

* This value for fluoride is based on an annual average daily air temperature between 12.1 and $14.6 \mathrm{C}$. 
APPENDIX 


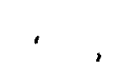




\section{PERFORMANCE STANDARDS FOR STATIONARY SOURCES}

Reference

Fossile Fuel St eam Generator
$42 \quad$ FR 37936 July 25,1977
St andards

Particulate: $\quad 0.10 \mathrm{lb} / 10^{6} \mathrm{BTU}$ Opacity: $\quad 20 \%$

$\mathrm{SO}_{2}: \quad 0.80 \mathrm{lb} / 10^{6} \mathrm{BTU}$

( 1 iquid fuel)

$1.21 \mathrm{~b} / 10^{6} \mathrm{BTU}$

(solid fuel)

$\mathrm{NO}_{2}$ :

$0.201 \mathrm{~b} / 10^{6} \mathrm{BTU}$

(gas fuel)

$0.301 \mathrm{~b} / 10^{6} \mathrm{BTU}$

( 1 iquid fuel)

$0.701 \mathrm{~b} / 10^{6} \mathrm{BTU}$

(solid fuel)

\section{WATER SAMPLE LOCATIONS}

Sample No.

OW $\sim W R-01$

OW-WR- 02

$O W-W R-06$

OW-WR- 08

OW-WR -16

OW-WR- 17

OW-WR-18

OW-WR- 19

$O W-W R-20$

OW-WR- 23

OW-WR -27

OW-WR -28

OW-WR -33

\section{Description of Location}

Pantex Lake

We 11 \#17

We $11 \# 6$

Retention Basin Playa

We $11 \# 20$

Sewage Discharge

We $11 \# 16$

Retention Basin Playa Test Well \#1

Zone 12 Test We 11 \#2

Drinking Water from Environmental Health, Bldg. 12-2

Sanitary Landfill playa

Drinking Water from 12-6 Cafeteria Irrigation Well at Bushland

Agricultural Experimental station 


\section{DISTRIBUTION}

No of Copies

DOE Albuquerque Operations Office/P. M. Ramey 19

DOE Amarillo Area Of $f$ ice/P. R. Wagner 2

DOE Technical Information Center $\quad 40$

Amarillo Bi-City County Health Department 1

Environmental Protection Agency, Region VI 1

Lawrence Livermore National Laboratory/A. J. Toy 1

Los Alamos National Laboratory/Wayne Hanson 1

Sandia National Laboratories - Albuquerque/W. D. Burnett 1

Texas Air Control Board 2

Austin, Texas/Cecil L. Bradford

Lubbock; Texas/Gerald Hudson

Texas Water Quality Board, District No. .1 1

Texas Department of Public Health 2

Aust in, Texas/Joseph Thiel

Canyon, Texas/Robert Adcock

Texas Department of Water Resources 2

Aust in, Texas/Robert Fleming

Amari11o, Texas/Mark Gates

Texas Park and Wildlife Department

Aust in/Charles D. Travis 1 